\title{
Transient Sources in Astrophysics: From Radio to Gamma-Rays
}

Meintjes P.J.*

Department of Physics, University of the Free State, PO Box 339, Bloemfontein, 9300, South

Africa

E-mail: MeintjpJ@ufs.ac.za / MeintPJ@gmail.com

The enhanced sensitivity of a new generation of telescopes, from radio (meerKAT-SKA) to VHE Gamma-Rays (CTA), may provide the required sensitivity to detect and study very faint accretion driven transient phenomena from a plethora of sources. In this study the physical magnetohydrodynamical processes that may influence plasma dynamics as well as drive particle acceleration and transient emission in some galactic accretion driven systems, more specifically the cataclysmic variables, are reviewed. The influence of magnetic fields on the dynamics of plasma, i.e. magnetic viscosity is described within the framework of turbulent magnetohydrodynamics and applied to explain episodes of transient mass transfer and accretion in cataclysmic variables. It is shown that magnetic fields influence the dynamics of plasma when the magnetic Hartmann number, $M \geq 1$. This may account for periods of enhanced mass accretion in disc accreting systems, and may explain the dwarf nova phenomenon in close binaries, as well as possible disc outflows and nonthermal emission. The role of magnetic fields is investigated in the propeller-driven mass outflow and accompanying non-thermal emission in the nova-like variable AE Aquarii from radio to Very High Energy (VHE) gamma-rays. The role that magnetic fields play in the possible non-thermal radio emission from polars is also discussed. Finally, the Fermi-LAT detection of gamma-ray emission from nova eruptions also open-up new possibilities to study particle acceleration and gamma-ray production in non-relativistic shocks. The fact that about 40-50 nova eruptions may occur in the Milky Way galaxy per year may provide a new class of gamma-ray source that can be studied in great detail with very sensitive future Cerenkov telescope arrays like the CTA.

$3 r d$ Annual Conference on High Energy Astrophysics in Southern Africa-HEASA2015,

18-20 June 2015

University of Johannesburg, Auckland Park, South Africa

${ }^{*}$ Speaker. 


\section{Introduction}

Most of the universe is in the form of plasma threaded by magnetic fields. Here one thinks invariably of the role magnetic fields play in the plasma dynamics of strongly magnetized compact objects like the magnetic cataclysmic variables (e.g. [1]), as well as disc accreting compact objets in X-ray binaries (e.g. [2], and references therein) and turbulent accretion discs to provide the required viscosity that can drive the observed mass accretion rates (e.g. [3]). In their seminal paper Shakura \& Sunyaev [3] quantified turbulent magnetohydrodynamic (MHD) viscosity $v_{\text {turb }}=$ $\alpha c_{\mathrm{s}} H$, where $\alpha<1$ is a dimensionless parameter which scales the magnitude of the viscosity in terms of the sound speed $c_{\mathrm{s}}$ and the disc scale height $H$. In terms of this model, the hysteresis S-curve characterizing a dwarf nova (DN) outburst in the $\Sigma-T_{\text {disc }}$ plane [ $\Sigma$ and $T_{\text {disc }}$ being the disc column density and temperature respectively] has been quantified in terms of a cyclic $\alpha$ parameter variation [4]. Simulated DN lightcurves resemble observed lightcurves most closely for values of $\alpha_{\text {cold }} \sim 0.01$ (low viscosity-quiescence) and $\alpha_{\text {hot }} \sim 0.1-0.3$ (high viscosity-outburst) [5].

A more quantitative description of accretion disc viscosity was provided with the detection of a Magneto Rotational Instability (MRI) in rotating magnetized fluids [6], which was applied to accretion discs (e.g. [7, 8, 9]). For example, these authors showed that the strong coupling between the disc plasma and weak disc magnetic fields via the Lorentz force results in the MRI being an effective mechanism to facilitate angular momentum transfer in accretion discs. This mechanism provides a more quantitative description of the viscosity variations between the "hot" and "cold" states of the accretion disc that characterize a DN outburst.

The role of magnetic fields in the mass transfer process in cataclysmic variables has been investigated (e.g. [10,11]). For example, Meintjes [10] showed that for Roche lobe mass transfer across a strong magnetic field near the $L_{1}$ region, the flow will essentially be decelerated to such an extent that the fluid (plasma) will accumulate behind the magnetic barrier leading to sporadic fragmented release and slingshot prominences. This may be the mechanism behind fragmented mass transfer in many cataclysmic variable systems. Furthermore it has been shown that magnetic fields play a very fundamental role in the magnetospheric propellering in disc accreting neutron star systems (e.g. [12], and references therein), as well as the cataclysmic variables, for example the nova-like variable AE Aquarii (e.g. [13, 14, 15, 16, 17, 18]).

Interest sparked in the magnetic cataclysmic variables, like AM Her, being potential nonthermal radio sources $[19,20,21,22]$. These authors showed that the lack of synchronization between the magnetized white dwarf and the secondary mass donor may induce strong field aligned potentials that can accelerate electrons to energies of the order of $1 \mathrm{MeV}$, resulting in synchrotron emission at radio wavelengths. Non-thermal radio outbursts have been detected from the nova-like variable system AE Aquarii [23] and modelled in terms of expanding synchrotron emitting clouds [24]. The effect is a self-absorbed $S_{v} \propto v^{\alpha}$ spectrum that extends to the infrared part of the spectrum [25]. This detection was the first indication that particle acceleration to relativistic energies can occur in a cataclysmic variable system. Subsequent detections of radio emission were made from non-magnetic and magnetic cataclysmic variables (e.g. [26]) which display a significant level of asynchronisity. The culmination of this must be AE Aquarii, which is probably the most asynchronous of all cataclysmic variables. Reports of Very High Energy (VHE) Gamma-ray emission from AE Aquarii have been made by two independent groups (e.g. [27, 28, 29]). Unfortunately 
these could not be verified by more recent campaigns (e.g. [30, 31, 32]). These examples, by far not comprehensive, provide evidence of the fascinating MHD processes driving accretion disc outbursts, propeller driven outflow, particle acceleration and non-thermal radio outbursts and possibly VHE gamma-ray emission in close binary systems like the cataclysmic variables.

In this study I am going to review some of the basic magnetohydrodynamics influencing the kinematics of plasmas and acceleration of particles to relativistic energies. I will then apply some of these principles to discuss the role of magnetic viscosity in disc accreting systems an attempt to provide a theoretical framework to evaluate episodes of transient emission or outbursts, like, for example, the DN eruptions or other transient accretion phenomena accreting systems. Then I will discuss possible non-thermal transient emission in the cataclysmic variable systems, focussing on the non-thermal emission in the nova-like variable $\mathrm{AE}$ Aquarii and the possibility of non-thermal radio emission in the magnetic cataclysmic variables (MCVs), using AE Her as an example. Lastly, I will briefly discuss the recent Fermi-LAT detections of gamma-ray emission form nova eruptions [33] which opens up a new frontier in gamma-ray astronomy since it presents a valuable opportunity to study particle acceleration and gamma-ray production in these events.

\section{Magnetohydrodyamics: Plasma Kinematics and Particle Acceleration}

\subsection{Plasma Kinematics}

In its most basic form the magnetic force density can be expressed in terms of two components, i.e

$$
\mathbf{f}_{\mathrm{m}}=\frac{B^{2}}{4 \pi R_{\mathrm{c}}} \mathbf{n}-\nabla_{\perp}\left(\frac{B^{2}}{8 \pi}\right)
$$

where the first term represents the magnetic tensile force towards the centre of curvature of a stressed hooped field line, while the second term represents the magnetic force as a result of the pressure gradient perpendicular to the field lines. Here $R_{\mathrm{c}}$ represents the radius of curvature of a stressed hooped field line, and $\mathbf{n}$ the normal vector pointing to the centre of curvature (e.g. [34]). This equation shows that hooped field lines can confine an enclosed plasma while the second component shows that magnetic fields can exert a pressure in the direction opposite to the magnetic field gradient, i.e. perpendicular to the direction of the field lines. It can be shown that the magnetic force has a significant influence on the dynamics of a conducting fluid, or plasma, i.e.

$$
\rho \frac{d \mathbf{v}_{\perp}}{d t}=\mathbf{f}_{\perp}-\frac{\sigma B^{2}}{c^{2}}\left(\mathbf{v}_{\perp}-\mathbf{w}\right),
$$

where $\mathbf{f}_{\perp}$ represents the local non-electromagnetic forces influencing the plasma flow and with $\mathbf{v}_{\perp}-\mathbf{w}$ representing the local relative velocity of the fluid relative to the frozen-in magnetic fields and where $\sigma$ and $B$ represent the plasma conductivity and magnetic field respectively [35]. Notice that the drift velocity ( $\mathbf{w})$ essentially determines the rate at which the magnetic field migrates relative to the particle population in the fluid (e.g. [35, 36]). This equation shows that high conductivity, combined with strong magnetic fields, have a significant influence on the motion of fluid across a magnetic field. There is essentially no, or very little, motion of fluid across the field as a result of the magnetic field being frozen into a highly conducting fluid and the magnetic pressure providing a resistance against a fluid element that attempts to cross it. This second term of the 
equation above essentially provides the foundation for magnetic viscosity. The effect of magnetic viscosity will be investigated in terms of the magnetic Hartmann $(M)$ and Reynolds $\left(R_{\mathrm{m}}\right)$ numbers [35] respectively. The Hartmann number is the ratio of magnetic stresses to particle viscosity in a magnetized fluid, while the Reynolds number essentially represents the ratio of the convection and diffusion associated with the magnetic field in the fluid. These two numbers are given respectively by

$$
\begin{aligned}
& M=\left(\frac{\sigma B^{2} L^{2}}{\mu c^{2}}\right)^{1 / 2} \\
& R_{\mathrm{m}}=\left(\frac{4 \pi L v \sigma}{c^{2}}\right),
\end{aligned}
$$

where $\sigma, \mu, B, L$ and $v$ represent the fluid electrical conductivity, the coefficient of dynamical viscosity, the magnetic intensity, the length scale of the field and the bulk flow speed respectively. For both the Hartmann and Reynolds numbers exceeding unity $\left(M>1 ; R_{\mathrm{m}}>1\right)$, the magnetic field will play an extremely important role in the dynamics of a magnetized fluid, which can have important consequences in astrophysical environments [37, 38].

\subsection{Particle Acceleration}

Apart from influencing bulk flow kinematics of a plasma, magnetic fields also play a fundamental role in particle acceleration processes [39] and subsequent non-thermal emission in astrophysical environments. It can be shown (e.g. [35]) that the electric and magnetic fields in a frame co-moving with a conducting fluid can be expressed in terms of the fields in the stationary laboratory frame, i.e.

$$
\begin{aligned}
\mathbf{E}^{\prime} & =\Gamma\left(\mathbf{E}+\frac{1}{c}(\mathbf{v} \times \mathbf{B})\right) \\
\mathbf{B}^{\prime} & =\Gamma\left(\mathbf{B}-\frac{1}{c}(\mathbf{v} \times \mathbf{E})\right),
\end{aligned}
$$

where $\left(\mathbf{E}^{\prime} ; \mathbf{B}^{\prime}\right),(\mathbf{E} ; \mathbf{B})$ represent the fields in the co-moving and laboratory frames respectively and where $\Gamma$ represents the bulk Lorentz factor. Here $\mathbf{v}$ represents the relative velocity between the fluid (co-moving) frame and the stationary (laboratory) frame. For a highly conducting fluid (i.e. $\sigma \rightarrow \infty$ ), the electric field in the co-moving frame $\mathbf{E}^{\prime} \rightarrow 0$ and field quantities transform as follows

$$
\begin{aligned}
& \mathbf{E}=-\Gamma\left(\bar{\beta} \times \mathbf{B}^{\prime}\right) \\
& \mathbf{B}=\Gamma \mathbf{B}^{\prime}
\end{aligned}
$$

where $\bar{\beta}=\frac{\mathbf{v}}{c}$. Notice that the electric field responsible for the acceleration of individual charged particles is tied to the bulk flow velocity ( $\mathbf{v})$ of the magnetized fluid with respect to the laboratory (stationary) reference frame. The maximum energy that can be attained by a charged particle $(q=Z e)$ can be determined by

$$
\begin{aligned}
\varepsilon_{\max } & =Z e \int \mathbf{E} . \mathbf{d s} \\
& =q \beta \Gamma R_{\mathrm{s}} B^{\prime}
\end{aligned}
$$




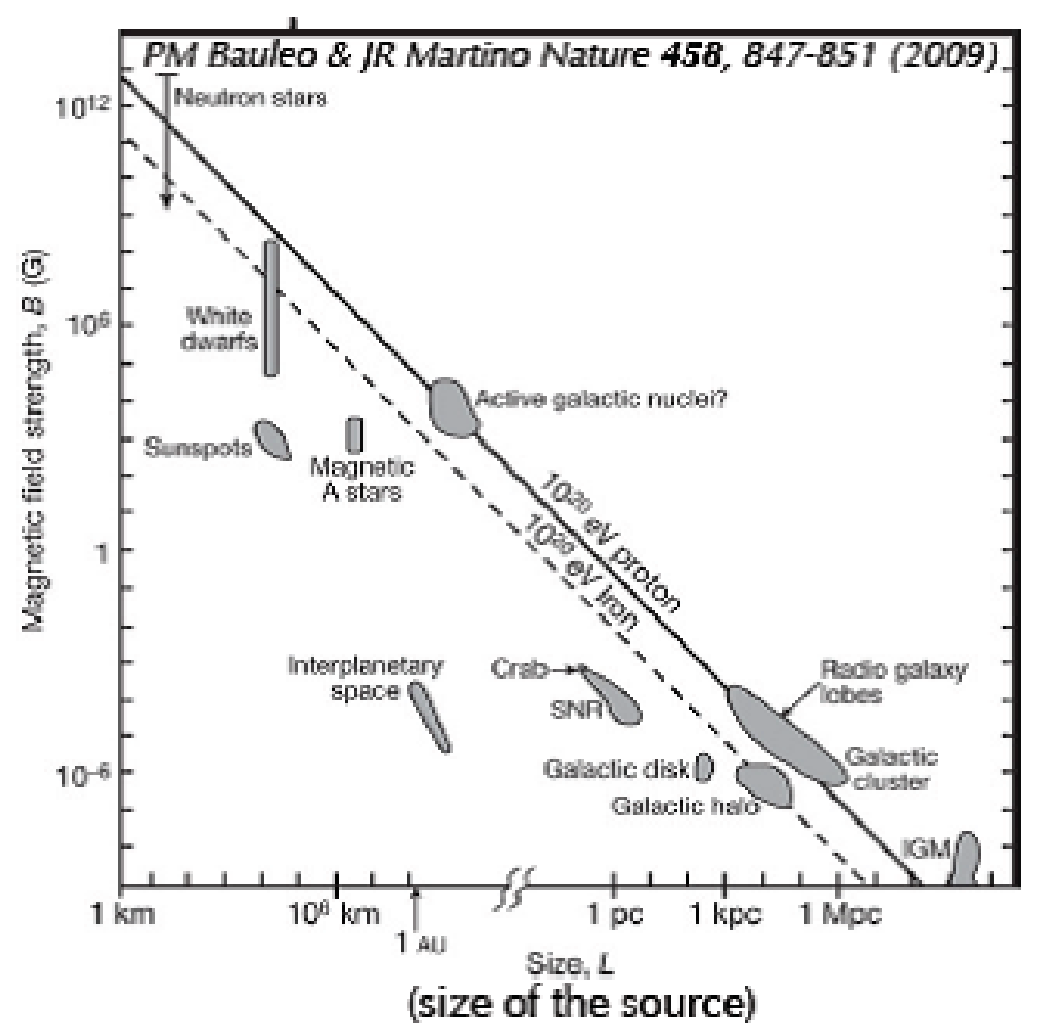

Figure 1: The Hillas Diagram. The horizontal axis represents the size of the source and the vertical axis the intrinsic source magnetic field strength. The diagonal lines correspond to the requirements for a $10^{20} \mathrm{eV}$ proton and heavy nucleus respectively. Adopted from [40].

where $\beta=(v / c)$ and $\Gamma$ represent the velocity and Lorentz factor respectively associated with the motion of the magnetized fluid with respect to a stationary observer. Here $R_{\mathrm{S}}$ and $B^{\prime}$ represent the size of the source and the magnetic field intensity in the frame of the source. This provides then an extremely attractive mechanism to accelerate charged particles in the magnetospheres of rapidly rotating, highly magnetized compact objects like neutron stars and white dwarfs. In this case the terms $\beta \Gamma$ will be associated with the rotational velocity of the magnetosphere of the compact object relative to a stationary observer. This expression clearly illustrates that for fast rotating objects $(\beta \Gamma>>1)$, the rotational kinetic energy reservoir relaxes the requirements on the strength of the intrinsic magnetic fields in cosmic sources to accelerate charged particles to high energies. It can be seen (Fig. 1) that even white dwarfs, with magnetic fields ranging between $\sim 10^{5} \mathrm{G}-10^{8} \mathrm{G}$, can produce charged particles of the order of $\varepsilon_{\max } \sim 10^{20} \mathrm{eV}$ (e.g. [40]).

This equation plays a fundamental role in the acceleration of particles in rapidly rotating magnetized compact objects (e.g. [41] and references therein). This essentially means that the field is in fact just the induced emf as long as there is relative motion between the source frame (co-moving) and the observer. A similar expression can be derived in the non-relativistic limit, by utilizing the 
magnetic induction equation [39], i.e.

$$
\frac{\partial \mathbf{B}}{\partial t}=\nabla \times \mathbf{v} \times \mathbf{B}-\nabla \times \eta \nabla \times \mathbf{B},
$$

where $\eta=\frac{c^{2}}{4 \pi \sigma}$ represents the resistivity of the plasma. This leads to two field components, perpendicular and parallel to the magnetic fields, i.e.

$$
\begin{aligned}
& \mathbf{E}_{\perp}=-\frac{\mathbf{v}}{c} \times \mathbf{B}\left[1+\left(\frac{1}{R_{\mathrm{m}}}\right)\left(\frac{L v}{c}\right)\left(\frac{\nabla \times \mathbf{B}}{\mathbf{B} \times \mathbf{v} / c}\right)\right] \\
& \mathbf{E}_{\|}=\left(\frac{1}{R_{\mathrm{m}}}\right) \mathbf{E}_{\perp} .
\end{aligned}
$$

As before, $R_{\mathrm{m}}$ represents the magnetic Reynolds number, which depends on the conductivity of the plasma, i.e. $R_{\mathrm{m}} \propto \sigma$ (e.g. [34]). Therefore it can be shown that for a higly conducting $(\sigma \rightarrow \infty)$ fluid, the equation for the perpendicular component reduces to the previous equation in the limit $\Gamma \rightarrow 1$, while the component of the electric field parallel to the magnetic field $\mathbf{E}_{\|} \rightarrow 0$. However, one can see that $\mathbf{E}_{\|} \neq 0$ for a fluid with finite conductivity. This component of the electric fields parallel to the magnetic fields is the driving force behind field-aligned currents (Birkeland-Dessler currents) flowing in magnetospheric circuits and $\mathbf{E}_{\|} \rightarrow \infty$ for regions where $R_{\mathrm{m}} \rightarrow 0$ (i.e. $\sigma \rightarrow 0$ ), i.e. for anomalously low conductivity. If the electron conduction velocity along the field exceeds the thermal velocity $\left(v_{\text {th }}=\left(\frac{3 k T_{\mathrm{e}}}{m_{\mathrm{i}}}\right)^{1 / 2}\right)$ of the particles, microinstabilities can develop (e.g. [39]), leading to anomalously low condictivity. This can be quantified in terms of the following expression [39]

$$
\frac{B \sigma}{n_{\mathrm{e}}} \geq 10^{2}
$$

with $n_{\mathrm{e}}$ the electron density, $\sigma$ the plasma electrical conductivity and $B$ the magnetic field. This condition shows that microinstabilities can develop where currents flow along strong magnetic fields where the electron density $\left(n_{\mathrm{e}}\right)$ is anomalously low.

A consequence of strong magnetic field aligned currents is the acceleration of particles in strong localized field aligned potential drops, or double layers (e.g. [42, 43]). These structures can have dimensions of the order of $\sim 100-1000 \lambda_{D e}$ (e.g. [44, 45]), with $\lambda_{\mathrm{De}}=7\left(T / n_{\mathrm{e}}\right)^{1 / 2}$ representing the Debye length of electrons in a plasma. Laboratory experiments seem to indicate that multiple double layers can be induced in a system [45] and that the size of the system essentially determines the size of these structures. In regions of anomalously low conductivity the currents will reach critical value, i.e. $j_{\|}=e n_{\mathrm{e}} f c_{\mathrm{s}}$, where $c_{\mathrm{s}}$ represents the speed of sound and where $n_{\mathrm{e}}$ is the electron density. Here $f \rightarrow 10$ [42] represents a critical limit for the trigger of microinstabilities that will increase the resistivity of the plasma. The potential drop along the field lines can be determined from energy conservation arguments by comparing the electromagnetic Poynting flux flowing into the double layer with the particle flux out of the double layer [42], i.e.

$$
\begin{aligned}
\Phi_{\|} & =\left(\frac{\text { em flux into double layer }}{\text { particle flux out of double layer }}\right) \\
& =\left(\frac{B^{2} v_{\mathrm{a}}}{8 \pi e n_{\mathrm{e}} f c_{\mathrm{s}}}\right),
\end{aligned}
$$


where $v_{\mathrm{a}}=B / \sqrt{4 \pi \rho_{\mathrm{e}}}$ represents the Alfvén velocity. An interesting consequence of double layer formation in regions where the electron density is extremely low is that the electric field become uncontrollably large, resulting in the total power to be released in terms of accelerated particles and possible associated high energy emission. The total power that can be released in accelerated particles is then (e.g. [43])

$$
\begin{aligned}
P_{\mathrm{dl}} & =\int \mathbf{j} \cdot \mathbf{E} d V \\
& =e n_{\mathrm{e}} c \Phi_{\|} d^{2}
\end{aligned}
$$

where c represents the speed of light and $d$ the width of the double layer.

Another particularly interesting mechansim for particle acceleration in magetized fluids or plasmas occurs where magnetic fields of opposite polarity merge (e.g. [39]), resulting in a global topological change of the magnetic field. It was originally proposed that magnetic null-points can be a site of plasma heating and particle acceleration (e.g. [46, 47, 48]). The first consistent steady state reconnection MHD model to illustrate how the current sheet collapsed into an X-point was developed independently by [49], [50] and [51], and is still known as the Sweet-Parker mechansism. Fast reconnection was proposed by [52], and a feature of this model was that the reconnection occurs over a very narrow region, in contrast to other models proposed where a current sheet developed over an extended region [53]. Particle acceleration in merging magnetic fields can be expressed very elegantly (e.g. [54, 43]) by considering the changing magnetic flux cutting through the orbit of a charged particle in the pre-merging fields, i.e.

$$
\frac{d \Phi_{\mathrm{B}}}{d t}=-\left(\frac{B \pi R_{\mathrm{L}}^{2}}{\tau_{\mathrm{m}}}\right)
$$

where $R_{\mathrm{L}}$ represents the Larmor radius of a spiralling charged particle and $\tau_{\mathrm{m}}=\left(R_{\mathrm{L}} / v_{\mathrm{m}}\right)$ represents the merging timescale. Here $v_{\mathrm{m}} \approx \alpha v_{\mathrm{a}}(\alpha \sim 0.1)$, where $v_{\mathrm{a}}$ represents the local Alfvén velocity (e.g. $[54,43])$. Utilizing Faraday's induction law the work done per unit charge (emf) in a single orbit [54] is

$$
e m f=\left(\frac{\pi B R_{\mathrm{L}} v_{\mathrm{m}}}{c}\right) .
$$

The electric field in the merging region (MR) is of the order of

$$
E_{\mathrm{MR}}=\left(\frac{\pi B v_{\mathrm{m}}}{c}\right)
$$

and must be of the same order as the field that drives a current in the dissipative region (DR), which is $E_{\mathrm{DR}}=(J / \sigma)$ [55]. In order to create a strong electric field, the conductivity must be anomalously low $(\sigma \rightarrow 0)$. As a charged particle accelerates in the field, the Larmor radius will increase. Ignoring for a moment energy losses, the maximum energy that can be attained occurs if the Larmor radius becomes comparable to the width of the current sheet, which is determined by Ampére' law, i.e.

$$
w=\left(\frac{c B}{4 \pi j}\right)
$$


where $j=e n_{\mathrm{e}} v_{\mathrm{d}}$ represents the current density in the diffusive region, where the drift speed $v_{\mathrm{d}} \sim f c_{\mathrm{s}}$ (with $c_{\mathrm{s}}$ representing the local sound speed), with $f \rightarrow 10$ representing the limit where microinstabilities will be excited that will further decrease the conductivity in the fluid (e.g. [54, 43]). The maximum energy to which electrons can be accelerated is then (e.g. [54, 43])

$$
\varepsilon_{\max }=\left(\frac{\pi \varepsilon e B v_{\mathrm{a}} w}{c}\right) .
$$

However, the real maximum energy will be determined by comparing the acceleration timescale, i.e. the time it will take the fields to merge in the diffusive, or merging region $\left(\tau_{\mathrm{a}}=w / v_{\mathrm{m}}\right)$, with a dominant energy loss mechanisms, for example synchrotron losses $\left(\tau_{\mathrm{s}}=2.37 \times 10^{-3} B^{2} \varepsilon_{\max }\right)$ (e.g. [43]). It has been shown [43] that the synchrotron loss time is several orders $\left(\sim 10^{4}\right)$ longer than the acceleration timescale for a $T \sim 10^{4} \mathrm{~K}$ plasma, with an electron density of $n_{\mathrm{e}} \sim 10^{10} \mathrm{~cm}^{-3}$ in a $B \sim 1000 \mathrm{G}$ field.

The purpose of the discussion in this section was to provide a very basic framework, or foundation, to evaluate the discussions to follow. In the following section an attempt will be made to provide an elementary model that may explain episodes of transient accretion in close binary systems, for example, the dwarf nova phenomenon. Then the attention will shift to particle acceleration and non-thermal emission over a wide frequency range in some cataclysmic variables.

\section{Magnetospheric Gating: The gravito-magnetic instability and disc outbursts}

The influence of magnetic fields on mass transfer and mass accretion in accretion driven systems have been investigated in great detail. For example, the observational properties of X-ray burst sources have been reviewed in great detail (e.g. [2]). Among the various transient accretion mechanisms discussed driving X-ray bursts depends on magnetsopheric gating (e.g. [56, 57, 2]). These models describe the transient accretion mainly in terms of episodic events of matter entering the magnetosphere of an accreting compact object as a consequence of large scale Rayleigh-Taylor instabilities (e.g. [56, 57]). Other transient phenomena in disc accreting systems is the dwarf novae events, which is now firmly associated with events of enhanced accretion rate as a result of a disc instability (e.g. $[58,59,60])$. As mentioned in the introduction, dwarf nova eruptions have been described elegantly in terms of a hysteresis S-curve in the $\Sigma-T_{\text {disc }}$ plane, which is driven by a cyclic disc viscosity variation [4] quantified by the MRI (e.g. [7, 8, 9]).

In this discussion, episodes of transient accretion in disc-fed systems is evaluated in terms of a gravito-magnetic instability (e.g. [37, 38]) as a result of a magnetic field build-up in the fluid which may trigger large scale Rayleigh-Taylor instabilities and episodic enhanced accretion that is associated with, among other, the dwarf nova eruptions. This magnetospheric gating effect $[10,11]$ may also be repsonsible for transient mass transfer episodes from secondary stars in close binaries. As pointed out in the previous section, the enhanced magnetic viscosity responsible for this phenomenon is quantified by the Hartmann number $M>1$ in a conducting fluid where the Reynolds number $R_{\mathrm{m}}>>1$.

For compact objects accreting matter from an accretion disc, it can be shown that for slow rotators, the ratio of the spin angular velocity of the rotating compact object $\left(\Omega_{*}=2 \pi / P_{*}\right)$ to the Keplerian velocity of the disc $\left(\Omega_{\mathrm{K}}=\left(G M / r^{3}\right)^{1 / 2}\right)$ inside the corotation radius satisfies $\Omega_{*} / \Omega_{\mathrm{K}}=$ 
$P_{\mathrm{K}} / P_{*}<1$, while for regions in the accretion disc outside the corotation radius $\Omega_{*} / \Omega_{\mathrm{K}}=P_{\mathrm{K}} / P_{*}>$ 1. In the event of magnetized disc accreting compact objects, the corotating magnetic field, presumably anchored in the disc will result in an outward magneto-centrifugal push [61] which effectively impedes the viscosity-driven radial inflow $[37,38]$. The effectivity of the outward magnetocentrifugal push of disc material outside the corotation radius depends on the magnetospheric strength of the compact accreting object, and the diffusivity of the magnetospheric field of the compact object frozen into the disc plasma. Based upon the magnetized disc around a slow rotator (e.g. [62, 34]) it can be shown [63] that this outward push in disc regions outside the corotation radius results in the radial inflow speed of a magnetized disc to be significantly less than for a non-magnetized Keplerian disc (see Fig. 2). It is believed that frozen-in magnetic fields will have a very significant influence on the dynamical properties of accretion disc plasma. It has been shown (e.g. [35]) that the flow of a conducting fluid across magnetic fields will result in a significantly modified flow profile. For example, [35] showed that for $M>>1$ the fluid rams into a magnetic obstruction too strong to advect, and plasma has to trickle across the magnetic obstruction with the typical $\mathbf{E} \times \mathbf{B}$ drift velocity. For $M<<1$ the magnetic field can be transported with the conducting fluid.

The build-up of disc material in a magnetized disc outside the co-rotation radius may lead to the disc becoming more turbulent, since the flow outside the corotation radius has departed from a purely Keplerian profile [63]. It can be shown that in the event of a turbulent disc, where the turbulent disc viscosity exceeds $v_{\mathrm{T}}=\alpha H_{\text {disc }} c_{s} \geq 10^{13}(\alpha / 0.1)\left(H_{\text {disc }} / 10^{8} \mathrm{~cm}\right)\left(c_{\mathrm{s}} / 10^{6} \mathrm{~cm} \mathrm{~s}^{-1}\right) \mathrm{cm}^{2} \mathrm{~s}^{-1}$ and magnetic diffusivity $\eta_{\mathrm{T}} \sim\left(c^{2} / 4 \pi \sigma_{\mathrm{T}}\right) \geq 10^{13} \mathrm{~cm}^{2} \mathrm{~s}^{-1}$ with frozen-in disc magnetic fields exceeding $B \sim 100 \mathrm{G}[37,38]$, the Hartmann number exceeds unity

$$
M>1\left(\frac{B_{\text {disc }}}{100 \mathrm{G}}\right)\left(\frac{L}{H_{\text {disc }}}\right)\left(\frac{v_{\mathrm{T}}}{10^{13} \mathrm{~cm}^{2} \mathrm{~s}^{-1}}\right)^{-1 / 2}\left(\frac{\sigma_{\mathrm{T}}}{10^{7} \mathrm{~s}^{-1}}\right)^{1 / 2} .
$$

With the disc magnetized to this level the gas will increasingly find the disc magnetic fields a barrier that will hamper the azimuthal Keplerian flow.

It has been shown $[37,38]$ that the effective gravity for disc matter orbiting the compact star with mass $M_{1}$ is

$$
\mathbf{g}_{\mathrm{eff}}=\left(-\frac{G M_{1}}{R^{2}}+\frac{v_{\phi}^{2}}{R}\right) \hat{\mathbf{R}} .
$$

From these equations $\mathbf{g}_{\text {eff }} \rightarrow 0$ for Keplerian flow, i.e. $v_{\phi} \rightarrow v_{\mathrm{K}}$, with $v_{\mathrm{K}}=(G M / r)^{1 / 2}$ representing the Keplerian velocity. However, one can see that if the azimuthal flow is decelerated across the fields, $\mathbf{g}_{\mathrm{eff}, \perp}<0\left(v_{\phi}<v_{\mathrm{K}}\right)$. This means that if the disc, or localized regions of the disc, has reached critical levels of magnetization, for which the Hartmann number $M>1$, the azimuthal flow will be decelerated such that the effective gravity in that region will point radially inward, which implies that during these states the disc plasma will experience a net inward pull towards the compact accreting object, resulting in the orbiting disc plasma spiralling inwards faster than when the motion was strictly Keplerian. This will result in a higher radial inflow and resultant accretion rate, which will manifest as an outburst or flare. Apart from the higher mass accretion rate, the rapid inflow from the disc, or localized regions of it, triggered by such a gravito-magnetic instability, has the potential to generate a turbulence field in the disc plasma. For a turbulent disc 


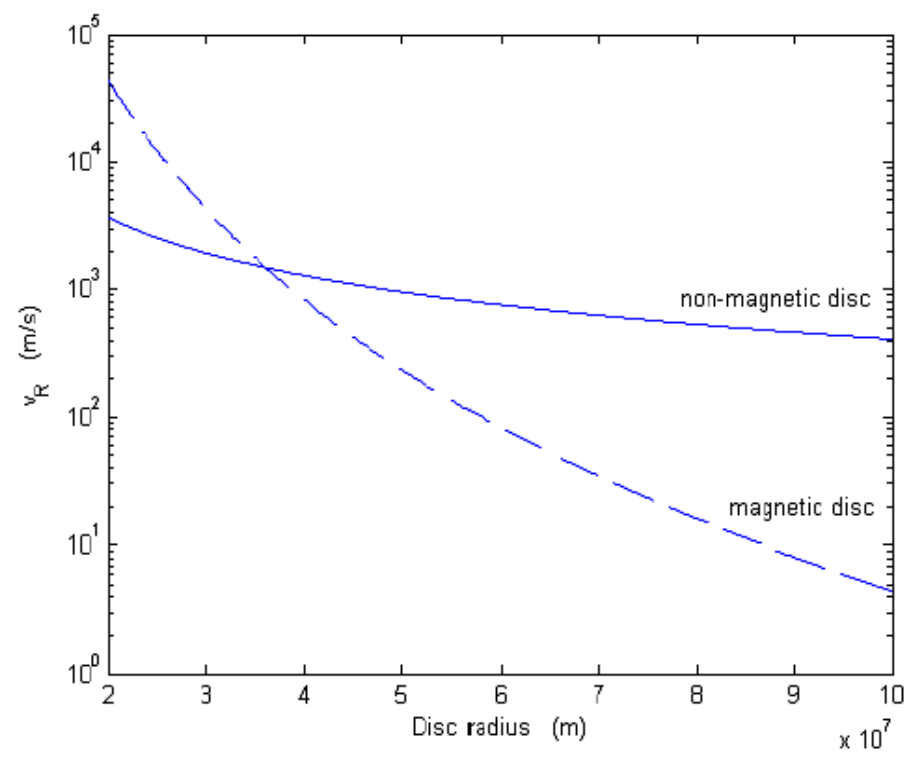

Figure 2: Radial dependence of the radial disc inflow speed $\left(v_{\mathrm{R}}\right)$ of a magnetized disc compared to a nonmagnetized accretion disc for $\mathrm{a} \sim 1 M_{\odot}$ white dwarf. Notice that outside the co-rotation radius the radial inflow speed for magnetized discs is noticeably lower. Adopted from [63].

with frozen-in magnetic fields of the order $B \sim 100 \mathrm{G}$ and density $\rho_{\text {disc }} \sim 10^{-8} \mathrm{~g} \mathrm{~cm}^{-3}$, it can be shown that the radial inward effective gravity is of the order

$$
\mathbf{g}_{\mathrm{eff}}=-3000\left(\frac{M_{1}}{M_{\odot}}\right)\left(\frac{R_{\mathrm{disc}}}{3 \times 10^{10} \mathrm{~cm}}\right)^{-2} \hat{\mathbf{e}}_{R} \mathrm{~cm} \mathrm{~s}^{-2}
$$

which is $\left|\mathbf{g}_{\text {eff }}\right|=3 \mathbf{g}_{\text {earth }}$ for the parameters chosen $[37,38]$. Therefore, the disc regions outside the corotation radius satisfying the conditions $M>1$ can spiral rapidly inwards and accrete onto the surface of the accreting compact object, resulting in a very high accretion luminosity.

It has been pointed out $[37,38]$ that this mechanism may be a possibility to explain the dwarf nova outbursts, and other transient mass accretion phenomena resulting in flares in accretion driven systems. The amount of matter and the timescale of the draining process depends obviously on the fraction of the disc that extends outside the corotation radius, as well as the fraction of that part of the disc being magnetically dominated. The model then can quantify the turbulent magnetic viscosity in terms of the state of magnetization of the disc, $\left(M_{\text {cold }}<1 ; M_{\text {hot }} \geq 1\right)$, which may be associated with turbulent viscosity, i.e. $\alpha_{\text {cold }} \sim 0.01 ; \alpha_{\text {hot }} \sim 0.1-0.3$.

It has been mentioned earlier that the magnetization of the disc above a critical limit $(M>1)$ is accompanied by mhd turbulence. Everywhere in the disc where the radial inflow speed exceeds the inflow speed normally associated with a Keplerian disc, turbulence will be generated. Hydrodynamical turbulence on the mean flow can be illustrated by breaking up the velocity in a mean $\left(\left\langle v_{i}\right\rangle\right)$ and fluctuating $\left(v_{i}^{\prime}\right)$ part (e.g. [64]). It can be shown that the rate of change of the kinetic 
energy of the mean flow due to the effect of turbulence can be expressed as

$$
\frac{\partial}{\partial t}\left(\frac{\left\langle v_{\mathrm{i}}\right\rangle\left\langle v_{\mathrm{i}}\right\rangle}{2}\right) \propto\left\langle v_{\mathrm{i}}^{\prime} v_{\mathrm{j}}^{\prime}\right\rangle \frac{\partial\left\langle v_{\mathrm{i}}\right\rangle}{\partial x_{\mathrm{j}}},
$$

where $\left\langle v_{\mathrm{i}}^{\prime} v_{\mathrm{j}}^{\prime}\right\rangle$ represents the so-called Reynolds stress, given by (e.g. [36], p. 173)

$$
\left\langle v_{\mathrm{i}}^{\prime} v_{\mathrm{j}}^{\prime}\right\rangle=-D_{\mathrm{T}}\left(\frac{\partial\left\langle v_{\mathrm{i}}\right\rangle}{\partial x_{\mathrm{j}}}+\frac{\partial\left\langle v_{\mathrm{j}}\right\rangle}{\partial x_{\mathrm{i}}}\right) \text {. }
$$

On the other hand, the energy equation concerning the fluctuating turbulent motion (e.g. [64]) is proportional to

$$
\frac{\partial}{\partial t}\left(\frac{\left\langle v_{\mathrm{i}}^{\prime}\right\rangle\left\langle v_{\mathrm{i}}^{\prime}\right\rangle}{2}\right) \propto-\left\langle v_{\mathrm{i}}^{\prime} v_{\mathrm{j}}^{\prime}\right\rangle\left(\frac{\partial\left\langle v_{\mathrm{i}}\right\rangle}{\partial x_{\mathrm{j}}}\right) .
$$

This indicates that turbulence results in the decrease of the mean kinetic energy of the smooth azimuthal flow, feeding it back to the turbulent eddies, i.e. turbulence generates more turbulence at the cost of the smooth mean flow. A property of turbulence (e.g. [36], pp. 158-175) is that large turbulent cells in the flow will divide into smaller cells, resulting in a Kolmogorov type energy cascade down to the shortest length scales. At these small scales, turbulent energy can be dissipated into heat. The cascade process accelerates towards shorter length scales (e.g. [65], pp. 132-133) as each eddy feeds its energy to shorter scales in a time that is a factor $2^{-2 / 3}$ shorter than the previous. This results in a total cascade time of the order of

$$
\tau_{\mathrm{cas}}=\left(\frac{l_{\max }}{v_{\mathrm{rms}}}\right) \text {, }
$$

where $l_{\max }$ and $v_{\mathrm{rms}}$ represent, respectively, the maximum length scale of the turbulent cells and the associated turbulent turnover velocity, typically of the order of a fraction of the local sound speed. Using the same parameters as above, it can be shown that the upper limit for the turbulence cascade time scale, i.e. the time for the largest possible turbulent perturbations with size $l_{\max } \sim H_{\text {disc }}$ and cascading speed $v_{\text {rms }} \sim \alpha c_{\mathrm{s}}[\alpha \sim 0.1]$ (e.g. [34], p. 43, 64), is of the order

$$
\tau_{\text {cas }} \sim 1000\left(\frac{l_{\max }}{H_{\text {disc }}}\right)\left(\frac{v_{\text {rms }}}{0.1 c_{\mathrm{s}}}\right)^{-1} \mathrm{~s}
$$

with $c_{\mathrm{S}} \sim 10^{6}\left(T / 10^{4} \mathrm{~K}\right)^{1 / 2} \mathrm{~cm} \mathrm{~s}^{-1}$. This cascade time scale represents the time delay before the energy contained in the turbulence manifests in heating of the gas, which is of the order of the dynamical timescale of the disc plasma. This will probably result in heating of the turbulent disc plasma in the event of the trigger of the gravito-magnetic instability. The result of this effect may be in the form of an inward spiralling heat-wave as the inward spiralling turbulent velocity fields decay into the dissipative regime, releasing the turbulent mehanical energy in the form of heat.

For a Kolmogorov type energy cascade of turbulent eddies of initial dimension $l_{\max , \mathrm{i}}$ and an average turn-over velocity $v_{\mathrm{rms}, \mathrm{i}}$ in the inertial range, to the dissipative scale where the corresponding values are $l_{\mathrm{d}}$ and $v_{\mathrm{rms}, \mathrm{d}}$ (e.g. [36], pp. 165-169), the energy dissipation rate per unit mass is

$$
\begin{aligned}
\varepsilon & =\left(\frac{v_{\mathrm{rms}, \mathrm{i}}^{3}}{l_{\mathrm{max}, \mathrm{i}}}\right) \\
& =\left(\frac{v_{\mathrm{rms}, \mathrm{d}}^{3}}{l_{\mathrm{d}}}\right) .
\end{aligned}
$$


Preserving the equality above between the inertial and dissipative regimes may imply a rather low turnover of turbulence into heating as a result of the low rms speed of the eddies in the dissipative regime. This may result in an accumulation of turbulent eddies (energy) in the disc with length scales comparable to the length scales in the dissipative regime (i.e. $l_{\mathrm{d}}$ ), which may be amplified by the mismatch between the inflow and outflow rate in the accretion disc in the build-up phase leading to the outburst. This reservoir of turbulence driven energy in the disc has to be released eventually. The thermalization of the gas during the DN outburst may be the direct result of the release of this reservoir of energy. The turbulence driven heat generation rate on the dissipative scale may be approximated by

$$
\begin{aligned}
\dot{u}_{\mathrm{d}} & =\rho\left(\frac{v_{\mathrm{rms}}^{3}}{l_{\mathrm{d}}}\right) \\
& =\rho v_{\mathrm{t}, \mathrm{d}}\left(\frac{v_{\mathrm{rms}}}{l_{\mathrm{d}}}\right)^{2} \operatorname{erg~cm}^{-3} \mathrm{~s}^{-1}
\end{aligned}
$$

where $v_{\mathrm{t}, \mathrm{d}}=v_{\mathrm{rms}} l_{\mathrm{d}}$ represents the coefficient of turbulent viscosity on the dissipative scale. It can be shown (e.g. [36], p. 166) that the size of the smallest eddies relates to the size of the largest according to

$$
l_{\mathrm{d}}=\left(\frac{R_{\mathrm{e}}}{R_{\mathrm{e}, \mathrm{crit}}}\right)^{-3 / 4} l_{\max },
$$

where $R_{\mathrm{e}}$ and $R_{\mathrm{e}, \text { crit }} \sim 100$ (e.g. [66], p. 47) represent respectively, the Reynolds number of the flow and the critical value where the size of the largest eddy equals the size of the smallest.

Since the typical Reynolds numbers of the flow may be of the order of $R_{e} \sim 10^{14}$ and the largest eddies probably have dimensions of the order of $H_{\text {disc }}=l_{\max } \sim 10^{8} \mathrm{~cm}$ (disc scale height), the smallest eddies dissipating turbulent energy in the gas is of the order

$$
l_{\mathrm{d}} \sim 0.1\left(\frac{R_{\mathrm{e}}}{10^{14}}\right)^{-3 / 4}\left(\frac{R_{\mathrm{e}, \mathrm{crit}}}{100}\right)^{3 / 4}\left(\frac{l_{\max }}{10^{8} \mathrm{~cm}}\right) \mathrm{cm} .
$$

The typical disc gas temperatures are probably of the order $T_{\text {disc }} \leq 10^{4} \mathrm{~K}$ (e.g. [60], p. 93), implying $v_{\text {rms }}=c_{\mathrm{s}} \leq 10^{6}\left(T / 10^{4} \mathrm{~K}\right)^{1 / 2} \mathrm{~cm} \mathrm{~s}^{-1}$, resulting in a coefficient of heat generating turbulent viscosity on the dissipative scale of

$$
v_{\mathrm{t}, \mathrm{d}} \leq 10^{6}\left(\frac{v_{\mathrm{rms}}}{c_{\mathrm{s}}}\right)\left(\frac{l_{\mathrm{d}}}{1 \mathrm{~cm}}\right) \mathrm{cm}^{2} \mathrm{~s}^{-1}
$$

For typical discs in cataclysmic variables with $\rho_{\text {disc }} \sim 10^{-8} \mathrm{~g} \mathrm{~cm}^{-3}$, the upper limit for the turbulence driven heat generation rate (when $v_{\mathrm{rms}} \rightarrow c_{\mathrm{S}}$ ) on the dissipative scale in the disc is of the order of

$$
\begin{aligned}
\dot{u}_{\mathrm{d}, \max } \sim & 10^{10}\left(\frac{n_{\mathrm{disc}}}{6 \times 10^{15} \mathrm{~cm}^{-3}}\right)\left(\frac{v_{\mathrm{t}, \mathrm{d}}}{10^{6} \mathrm{~cm}^{2} \mathrm{~s}^{-1}}\right) \\
& \times\left(\frac{v_{\mathrm{rms}}}{c_{\mathrm{s}}}\right)^{2}\left(\frac{l_{\mathrm{d}}}{1 \mathrm{~cm}}\right)^{-2} \mathrm{erg} \mathrm{cm}^{-3} \mathrm{~s}^{-1} .
\end{aligned}
$$


This dissipation rate is sufficient to make the disc a significant contributor to the radiation in accretion driven systems. For example, it can be shown that for thin accretion discs $\lambda_{\text {disc }}=(H / R) \sim 10^{-2}$ (e.g. [60], p. 94), where $H \sim 10^{8} \mathrm{~cm}$, the total disc luminosity in a volume corresponding to a fraction $\beta \sim 0.1$ of the turbulent disc is of the order

$$
\begin{aligned}
L_{\mathrm{disc}, \max } \approx & \dot{u}_{\mathrm{d}, \max }\left(\beta R_{\mathrm{disc}}\right)^{2} H \\
\leq & 10^{35}\left(\frac{\dot{u}_{\mathrm{d}, \max }}{10^{10} \mathrm{erg} \mathrm{cm}^{-3} \mathrm{~s}^{-1}}\right)\left(\frac{\beta}{0.1}\right)^{2} \\
& \left(\frac{R_{\mathrm{disc}}}{5 \times 10^{9} \mathrm{~cm}}\right)^{2}\left(\frac{H}{10^{8} \mathrm{~cm}}\right) \mathrm{erg} \mathrm{s}^{-1} .
\end{aligned}
$$

This result shows that turbulence dissipation in $\beta \sim 0.1$ of the disc can power the dwarf novae. This luminosity is completely consistent with the inferred disc luminosities of $L_{\mathrm{disc}} \sim 10^{34} \mathrm{erg} \mathrm{s}^{-1}$ of the systems U Gem, SS Cyg, Z Cha and VW Hyi during DN outbursts (e.g. [67], p 214 and references therein).

It has been shown that draining of the accretion disc outside the corotation radius in the event of a magneto-gravitational instability could be associated with episodes of enhanced mass accretion onto a compact object, which can explain, among other, the dwarf nova events. For accreting systems where the gravito-magnetic instability is triggered in the presence of the ambient magnetospheric field of the compact object, it may result in the inward advection of the magnetospheric field anchored in the disc. This will lead to a deformation of the large scale magnetospheric field, with the associated outward directed centrifugal acceleration of plasma trapped in the disc corona [68]. These authors have shown that outward centrifugal acceleration along the open field lines will be triggered for magnetospheric fields anchored in the disc with angles $i \leq 60^{\circ}$. During events where the disc would drain, magnetic fields frozen into the disc may advect with the fluid, resulting in magnetic reconnection, particle acceleration and the centrifugal ejection (e.g. [69], [48]) of magnetized synchrotron emitting bubbles (see Fig. 3) from the system (e.g. [70, 24]) that may be detectable with new generations of radio telescopes like meerKAT, the SKA precursor currently under construction in South Africa. This telescope will consist of an array of 64 telescopes, each with a $\sim 13.5 \mathrm{~m}$ reflector dish diameter, equipped with very sensitive UHF-band (0.55-1.015 GHz), L-Band ( 0.9-1.67 GHz) and X-band (8-14.5 GHz) receivers (https://en.wikipedia.org/wiki/MeerKAT).

\section{Non-Thermal Emission in Cataclysmic Variables}

The initial detection of radio emission from $\operatorname{AE}$ Her $[19,20,21,22]$ and the strong nonthermal radio outbursts from the nova-like variable AE Aquarii [23, 24] sparked huge interest in cataclysmic variables, as possible sources of non-thermal emission. It has been shown by Mason \& Gray [26] that the level of the radio emission from some polars and intermediate polars correlates with the asynchronisity of the white dwarf rotation with respect to the binary period: The higher the asynchronisity, the stronger the radio emission. 

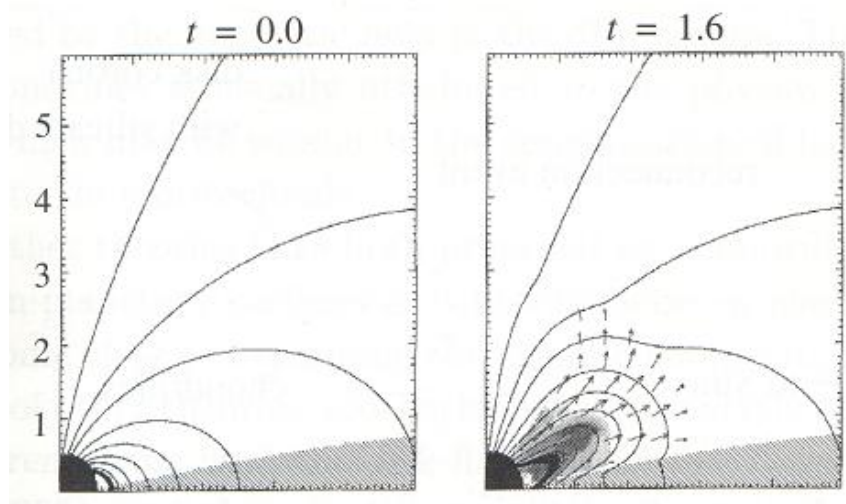

$\log T$
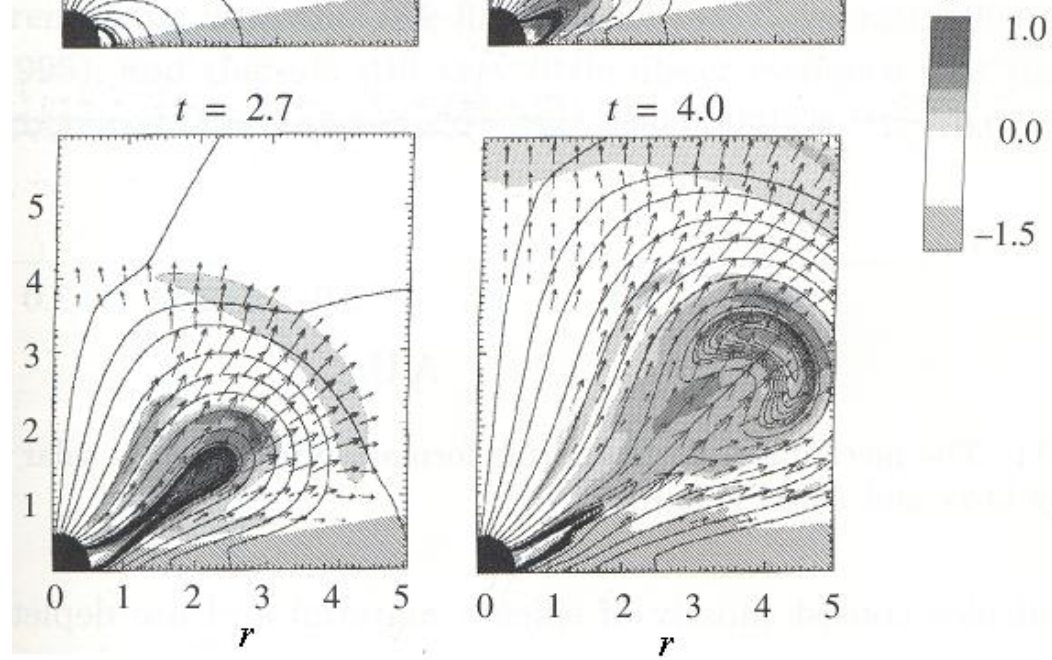

Figure 3: Centrifugal ejection of magnetized plasma bubbles from accretion discs. The grey scale on the right represents the coronal temperature in the flux tubes in relation to the steady state disc temperature. Adopted from [69], [48].

\subsection{AE Aquarii: A Rapidly Asynchronous Propeller Driven Pulsar}

The enigmatic multi-wavelength properties of the novalike variable star AE Aquarii have resulted in it being probably one of the best studied and documented sources in the sky. The source displays transient emission from radio wavelengths up to possible burst-like VHE gamma-rays (e.g. [71, 72] for reviews). The source consists of a rapidly rotating $\left(P_{*}=33 \mathrm{~s}\right)$ highly magnetized white dwarf $\left(B_{*} \sim 10^{6} \mathrm{G}\right)$ orbiting a K3-4 secondary star every $P_{\text {orb }} \sim 10$ h (e.g. [72] and references therein). This makes the white dwarf in AE Aquarii one of the most asynchronous rotators among the cataclysmic variables ([13]). A pulse timing study of the $33 \mathrm{~s}$ rotation period of AE Aquarii [14] shows that the white dwarf is spinning down at a rate of $\dot{P} \sim 5 \times 10^{-14} \mathrm{~s} \mathrm{~s}^{-1}$, implying a spin-down luminosity of the order of $P_{\mathrm{sd}} \sim 10^{34} \mathrm{erg} \mathrm{s}^{-1}$, which exceeds the accretion luminosity $L_{\text {acc }} \sim 10^{31} \mathrm{erg} \mathrm{s}^{-1}$ inferred from HST UV and other X-ray observations, by a factor of $\sim 1000$ (e.g. [71]). This implies that the white dwarf in AE Aquarii is in a propeller phase, with very little material accreting onto the surface of the white dwarf. This places the white dwarf in $\mathrm{AE}$ Aquarii in the same category as the rotational power pulsars, which shows a similar ratio of $\mathrm{X}$-ray luminosity to spin-down power (e.g. [73, 74]). From a model independent energetics point 


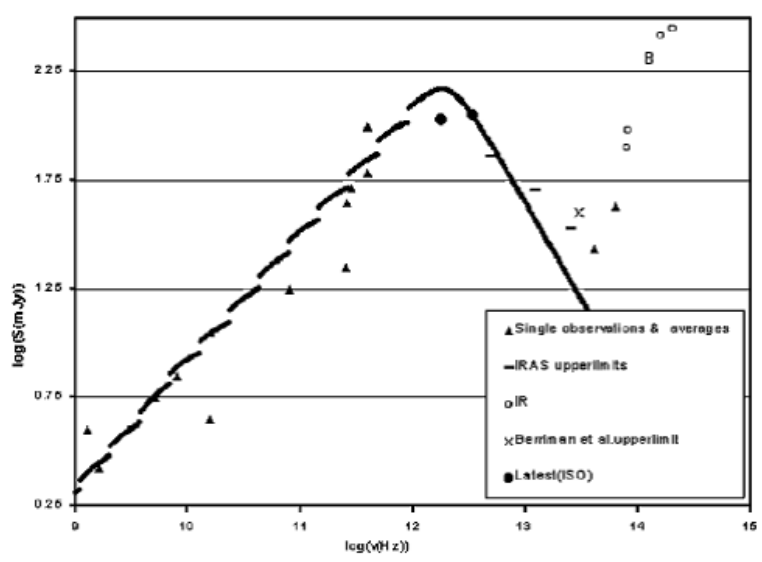

Figure 4: Superposition of a few synchrotron emitting flares with high internal magnetic fields of $B>$ $1000 \mathrm{G}$ and electron energies of the order of $\varepsilon_{\mathrm{e}} \sim 1 \mathrm{MeV}$. Adopted from [76].

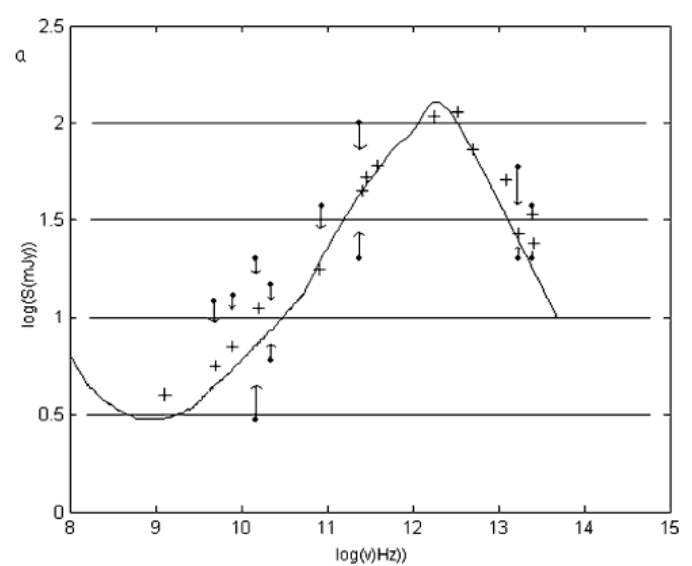

Figure 5: The superposition of several blobs with average field of $B \sim 400 \mathrm{G}$ and average electron energy of the order of $\varepsilon_{\mathrm{e}} \sim 20 \mathrm{MeV}$. Adopted from [77].

it can be shown that the fast rotation period, combined with an inferred magnetic field strength of $B_{*} \sim 10^{6} \mathrm{G}$ based on circular polarization measurements (e.g. [72] and references therein), provide the required potential (e.g. see Fig. 1) to accelerate charged particles in the white dwarf magnetosphere $\left(R \sim 10^{8} \mathrm{~cm}-10^{10} \mathrm{~cm}\right)$ to energies of the order of $\varepsilon_{\max } \sim 10^{20} \mathrm{eV}$.

The presence of a particle accelerator in the AE Aquarii (e.g. [75]) system was confirmed with its detection in radio frequencies in the late 1980s [23, 24]. The emission is highly transient and non-thermal, with a brightness temperature exceeding $T_{\mathrm{b}}>10^{10} \mathrm{~K}[23,24]$. The transient radio emission can readily be explained in terms of a superposition of expanding synchrotron emitting plasma clouds, which cool as they expand [70, 24]. The characteristic signature of this spectrum is $S_{v}(\mathrm{mJy}) \propto v^{\alpha}$, with $\alpha \sim(0.3-0.5)$ ([24]), and extends up to frequencies in the Spitzer telescope range $(v \sim 2000 \mathrm{GHz})$ [25]. Detailed modelling of the non-thermal radio outbursts (see Fig. 4 and Fig. 5) have been performed and presented [76, 77] based on two independent models.

Both these models were based on the synchrotron emission from relativistic electrons in magnetized clouds. The first attempt to model non-thermal radio emission from AE Aquarii [76] considered the synchrotron emission from magnetized bubbles in the mass transfer stream from the secondary star (Fig. 4), which are pumped by the rapidly rotating white dwarf magnetic field, in a betatron proceses (e.g. [39]), while the second attempt focussed on the ejection of magnetized clouds through a Kelvin-Helmholtz instability as the magnetosphere periodically sweeps across the mass transfer stream from the secondary star (Fig. 5). In this model the acceleration process is believed to be magnetic reconnection, discussed earlier. Both these attempts to model the non-thermal radio-IR spectral energy distribution (SED) show that the synchrotron emission in expanding synchrotron emitting clouds can account for the observed emission in this frequency regime.

The non-thermal nature of the radio-IR synchrotron flares from AE Aquarii resulted in it being classified as a low-power analogue to the enigmatic Cyg X-3 [24], and sparked huge interest for further follow-up studies at higher energies. AE Aquarii has been studied extensively in the VHE 


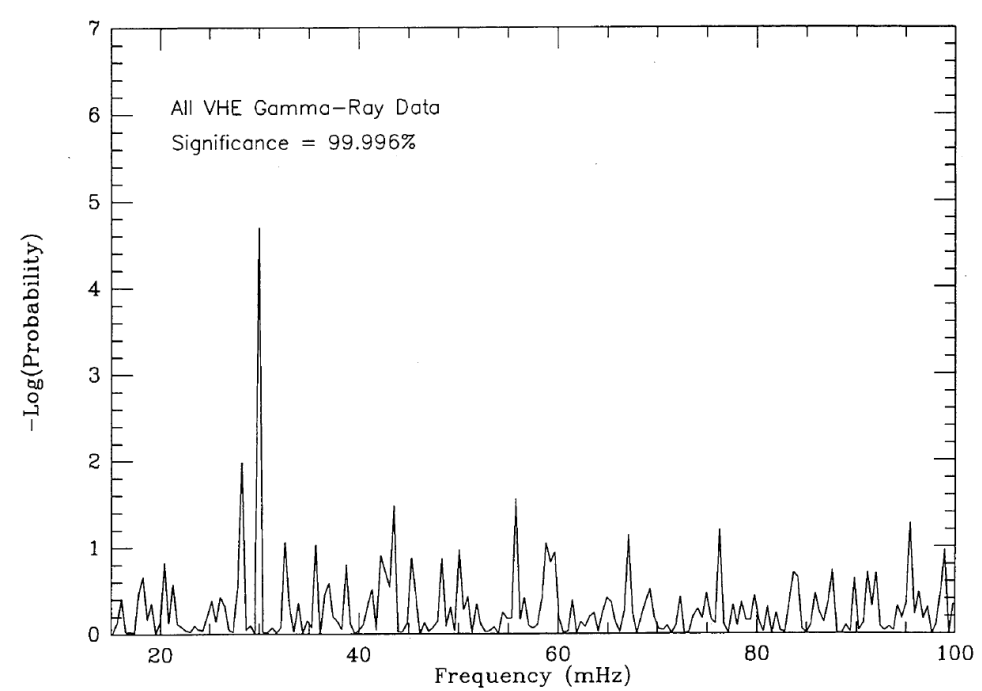

Figure 6: Pulsed TeV gamma-ray emission from AE Aquarii at a period close to the spin period of the white dwarf utilizing the total data set spanning 4 years. Adopted from [28].

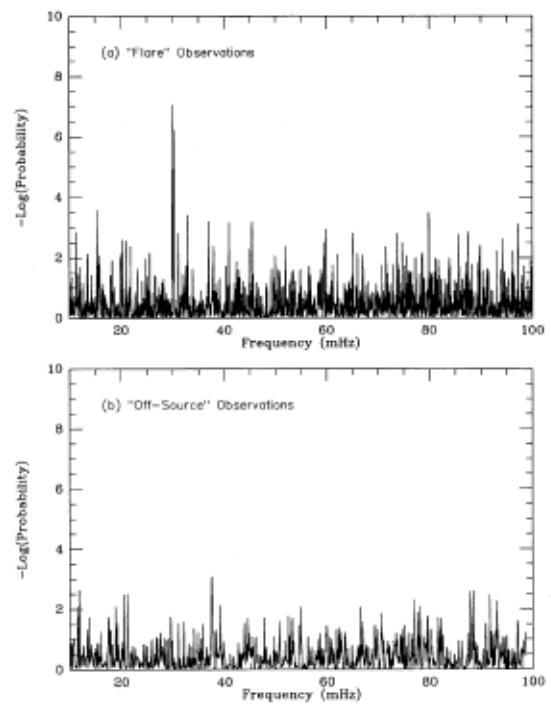

Figure 7: Pulsed TeV gamma-ray emission from AE Aquarii during periods of enhanced activity. Adopted from [28].

regime in the late 1980s and 1990's by two independent groups with telescopes at Nooitgedacht (South Africa) and Narrabri (Australia) respectively (e.g. [27, 28, 29]). Between 1988 and 1991 the Nooitgedact Cerenkov array has observed AE Aquarii for $\sim 240$ hours on moonless nights and registered $\sim 233600$ Cerenkov events [28]. By utilizing the complete dataset spanning 4 years, pulsed emission have been detected very close to the spin period of the white dwarf at the $99.996 \%$ significance level [28](e.g. Fig. 6).

Optical photometry that was performed simultaneously with the VHE observations at Nooitgedacht, utilizing telescopes at the South African Astronomical Observatory (SAAO), were used to extract the VHE gamma-ray observations where the source displayed flaring activity. These observations were analysed for periodicity and compared to comparison "off-source" observations. This showed that the pulsed VHE emission is associated with periods of enhanced activity of the source (e.g. Fig. 7). Very significant pulsed emission very close to the white dwarf spin period was also detected during a single observation at the 99.999\% significance level [28] (see Fig.8).

Apart from this consistent pulsed emission close to the spin period of the white dwarf, burst like emission have been detected by both VHE gamma-ray groups [27, 29], and displayed in Fig. 9 (Nooitgedacht) and Fig 10 (Narrabri). During the night of the Nooitgedacht observations, simultaneous optical observations were made utilizing the $0.75 \mathrm{~m}$ telescope at SAAO. It can be seen that the source showed significant optical variablilty during that particular night. The bottom panel of Fig 9 showed the photomultiplier tube currents of the three operational Nooitgedacht Cerenkov telescopes to show that the $\mathrm{TeV}$ burst was not as a result of instrumental instability. Both these burst showed double peaked pulsed emission when folded with the $P_{*}=33.08 \mathrm{~s}$ white dwarf period, implying that both poles of the rotating white dwarf is visible in VHE gamma-rays [27, 29].

The nature of the non-thermal emission from AE Aquarii, from radio to VHE gamma-rays, have been investigated in detail by [75], [43] and [78]. It was shown that episodes of particle 


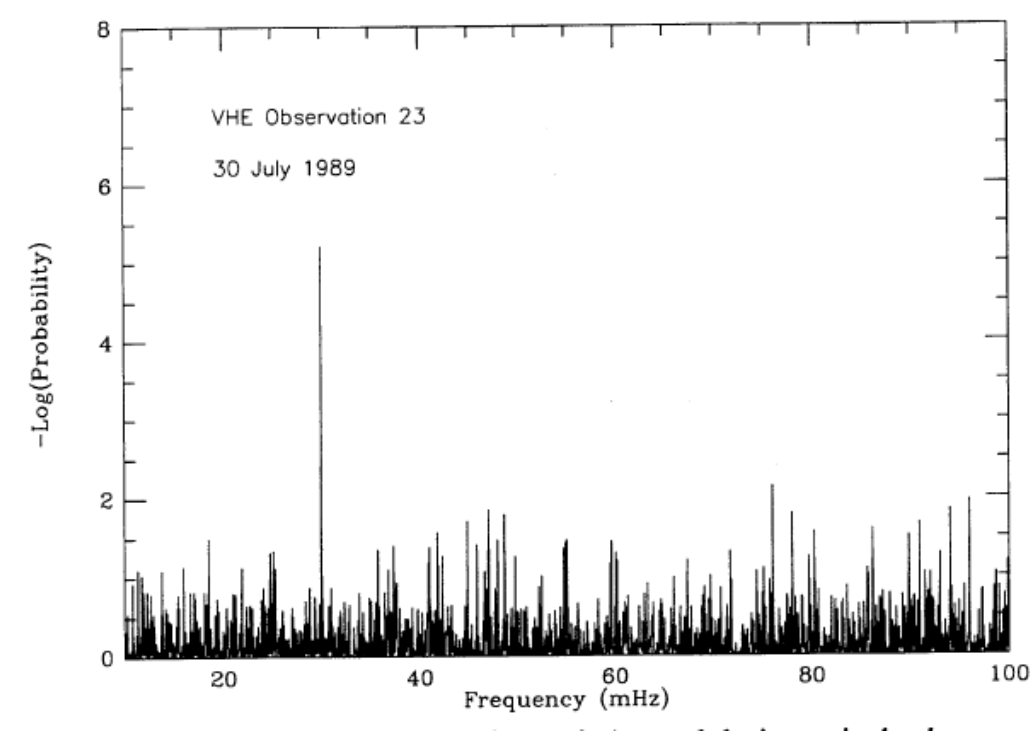

Figure 8: Pulsed TeV gamma-ray emission from AE Aquarii during a single observation close to the spin period of the white dwarf. Adopted from [28].
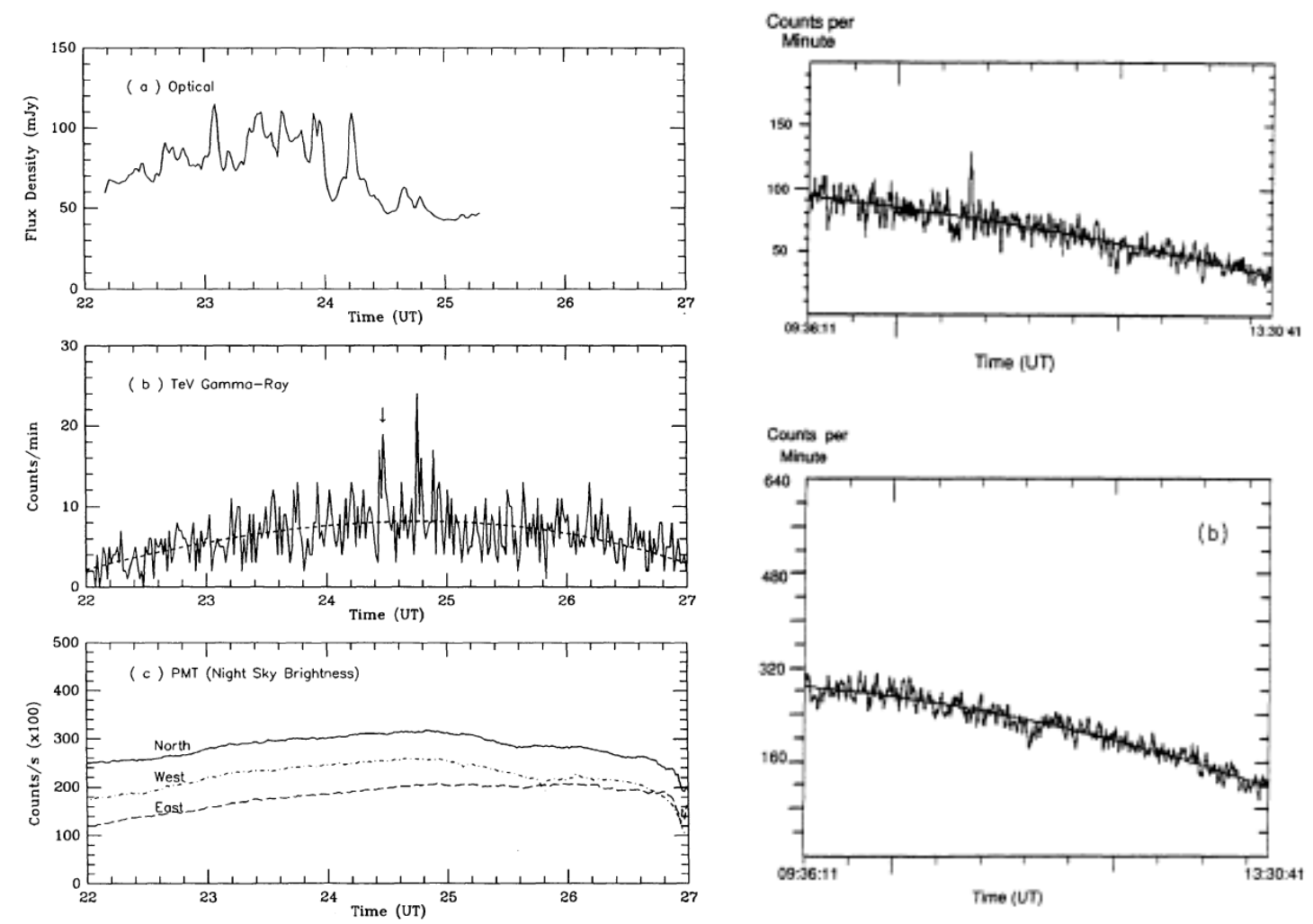

Figure 9: Burst-like $\mathrm{TeV}$ gamma-ray emission from $\mathrm{AE}$ Aquarii observed with the Nooitgedacht Cerenkov telescope in South Africa. Adopted from [29].

Figure 10: Burst-like VHE gamma-ray emission from AE Aquarii observed with the Cerenkov telescope in Narrabri. Adopted from [27]. 
acceleration may be associated with the propeller process in AE Aquarii. Magnetic shear in the ejection region will induce field-aligned currents in the magnetosphere. Huge potential differences (double layers) can be generated in the circuit where the gas density becomes very low. This will occur close to the white dwarf. In this region potentials of the order of $\Phi \sim 300 \mathrm{TV}$ (TV = teravolt) can be generated. Synchrotron losses on electrons close to the white dwarf will restrict them to energies of the order of $\mathrm{E}<50 \mathrm{keV}$. As protons and ions are not affected by synchrotron losses, energies in excess of $1 \mathrm{TeV}$ are possible. Very high-energy (VHE) gamma-ray emission can be produced if these proton and ion beams collide with the clumpy gas in, or outside, the ejection zone. We show that pulsed VHE gamma-ray emission is possible, if the gas particle density of the target matter is at least $N_{\mathrm{g}} \sim 10^{13} \mathrm{~cm}^{-3}$, which will be satisfied for typical gas particle densities $N_{\mathrm{g}} \sim 10^{16} \mathrm{~cm}^{-3}$ of the blobs inside the clumpy accretion stream from the secondary star. We show that the VHE gamma-ray bursts that were reported by two groups can be explained in terms of an exploding double layer, which will result in a cataclysmic burst of VHE protons and ions over short time-scales. The total power released in these burst can be $P_{b} \sim 10^{34} \mathrm{erg} \mathrm{s}^{-1}$, which is equal to the spin-down power of the white dwarf, and is enough to explain the reported VHE gamma-ray bursts. This implies that the whole reservoir of spin-down power has gone into the production of VHE particles and gamma-ray emission.

More recently a study of the properties of the hard X-ray $(\varepsilon>10 \mathrm{keV})$ pulsations of $\mathrm{AE}$ Aquarii detected in the Suzaku data [74], revealed that AE Aquarii displays the same ratio $\left(\sim 10^{-4}\right)$ of hard X-ray luminosity $\left(L_{\mathrm{X}} \sim 10^{30} \mathrm{erg} \mathrm{s}^{-1}\right)$ to spin-down power $\left(P_{\mathrm{sd}} \sim 10^{34} \mathrm{erg} \mathrm{s}^{-1}\right)$, which is similar to the relationship normally observed for young spin-powered pulsars [73]. Furthermore, the Suzaku hard X-ray spectrum seems to suggest a power-law shape, with a photon index of $\alpha \sim 2.3$ [74], indicative of a non-thermal emission process. The pulsar-like acceleration of particles in the white dwarf magnetosphere in AE Aquarii has been investigated by [71] and [78]. It has been shown that huge potentials of the order of $V \sim 3 \times 10^{12} P_{33}^{-2} \mu_{33}$ Volt can exist between the white dwarf surface and the light cylinder, which can accelerate charged particles to VHE-TeV energies. Here $P_{33}$ and $\mu_{33}$ represent the white dwarf spin period (33 s) and the magnetic moment of the white dwarf $\left(\sim 10^{33} \mathrm{G} \mathrm{cm}^{3}\right)$ respectively. Keeping in mind synchrotron losses, these authors showed that relativistic electrons with Lorentz factors of the order of $\gamma \sim 10^{5}$ can be produced still inside the light cylinder of the white dwarf, and will radiate synchrotron radiation in the magnetospheric field $\left(B_{\mathrm{lc}} \sim 100 \mathrm{G}\right)$ in the proximity of the light cylinder at frequencies $v \sim$ few $\times 10^{18} B_{100} \gamma_{5}^{2} \mathrm{~Hz}$, where $B_{100}$ and $\gamma_{5}$ represent the magnetic field $(\sim 100 \mathrm{G})$ and Lorentz factor $\left(\sim 10^{5}\right)$ respectively. It was further shown that the inverse-Compton scattering of $\gamma \sim 10^{5}$ electrons on optical photons from the seconday star $\left(\varepsilon_{\mathrm{ph}}<5 \mathrm{eV}\right)$, provide interesting possibilities for the production of gamma-rays with energies of the order $\varepsilon_{\gamma} \leq 0.2 \gamma_{5}^{2}\left(\varepsilon_{\mathrm{ph}} / 5 \mathrm{eV}\right) \mathrm{TeV}$, which would place it in the Fermi-LAT energy range [71,78].

\subsection{Non-Thermal Radio Emission from Polars}

Radio emission was detected from the polar AM Her in 1981 by [19], with an inferred brightness temperature $T_{\mathrm{b}} \geq 10^{9} \mathrm{~K}$ for a source radius of the order of $10^{11} \mathrm{~cm}$ and a distance of $d \sim 100 \mathrm{pc}$, that could imply a possible non-thermal origin. It was interpreted as gyrosynchrotron emission of $\sim 0.5 \mathrm{MeV}$ electrons radiating at the 40 th harmonic in a region where the white dwarf magnetic field was about $\sim 40$ Gauss. The emission declined over time, but the occurence of a flare in July 
1982 where the flux level increased from approximately $0.55 \mathrm{mJy}$ to $9.7 \mathrm{mJy}$, a factor of $\sim 20$ increase, which was $100 \%$ right circularly polarized, indicated that it was possibly the result of a coherent process, possibly a cyclotron-maser operating near the surface of the secondary star (e.g. [22] for a review) where the magnetic field was $\sim 1000$ Gauss. Therefore, it appears as if AM Her have two distinct varieties of radio emission.

The presence of electrons with energies of the order of $\sim 0.50 \mathrm{MeV}$ is still unclear, but a few possibilities can be investigated. The basis for the electrodynamic acceleration of charged particles in rotating magnetospheres is the differential motion between the magnetized fluid in the source frame and the observer. A high level of differential rotation usually corresponds to the level of asynchronisity of the compact object's rotation with respect to the orbital motion. It is believed that the high magnetic fields of the white dwarfs in the AM Her type systems, i.e. the polars, is strong enough to phase lock the white dwarf rotation with the rotational period of the secondary star, which is synchronized with the binary (e.g. [1, 34, 67]). However, by using the JupiterIo system as an example, it has been shown [79] that small departures from synchronization can result in huge field aligned potentials that can be induced along the magnetic flux tubes connection the Jupiter-Io system. This concept was adopted and applied to the phase locked magnetospheric connection between highly magnetized white dwarfs and the secondary mass donor in AM Her, the prototypical polar [20]. By using Eqs 2.6 and 2.7 one can show that a potential difference can be generated between the primary white dwarf and secondary mass donor, as a result of an asynchronisity in the rotation between the white dwarf and secondary star, which is phase locked with the binary period, given by $\Delta \beta_{\phi}=\frac{1}{c}\left(v_{\phi, w d}-v_{\phi, b}\right)$, which results in

$$
\varepsilon_{\max }=q \Delta c \beta_{\phi} B^{\prime} L
$$

where $B^{\prime}$ represents the dipolar magnetic field strength of the white dwarf at the distance of the secondary star, which is $d \sim 70 R_{*}$, with the potential difference estimated across a length scale $L$ that corresponds to the diameter of the secondary star $L \sim 6 \times 10^{10} \mathrm{~cm}[20]$. For AM Her the radius of the white dwarf is approximately $R_{*} \sim 10^{9} \mathrm{~cm}$, then $L \sim 60 R_{*}$, and the surface field strength is approximately $B_{*} \sim 2 \times 10^{7}$ Gauss (e.g. [20]). It can be seen that even a small departure in synchronisity of the order of $\Delta \beta_{\phi} \sim 10^{-9}$ can result in potentials that can accelerate charged particles like electrons to energies of the order

$$
\varepsilon_{e, \text { max }} \sim 1\left(\frac{\Delta \beta_{\phi}}{10^{-9}}\right)\left(\frac{B_{*}}{2 \times 10^{7} \mathrm{G}}\right)\left(\frac{R_{*}}{10^{9} \mathrm{~cm}}\right)^{3}\left(\frac{d}{70 R_{*}}\right)^{-3}\left(\frac{L}{60 R_{*}}\right) \mathrm{MeV}
$$

in field aligned potentials between the magnetic white dwarf and secondary star. It has been mentioned earlier that regions of anomalously low conductivity can produce "spark gaps" or double layers where particles like electrons can be accelerated to high energies.

However, the magnetic connection between the magnetospheres of the white dwarf and secondary star may also result in magnetic reconnection, where particles like electrons can be accelerated. The magnetic properties of secondary stars in close binaries have been investigated by applying several dynamo models that can drive the magnetic properties of these stars [10,11]. It has been shown that the secondary stars in cataclysmic variables with binary periods between $P_{\text {orb }} \sim 3 \mathrm{~h}-7 \mathrm{~h}$ can sustain large scale surface fields with polar values between $B_{\circ, 2} \sim 2900 \mathrm{G}-3300 \mathrm{G}$ [11]. In 
fact the following empirical relation was derived for the surface polar field

$$
B_{\circ, 2}=\left[112\left(\frac{P_{\text {orb }}}{h}\right)+2530\right] \mathrm{G} .
$$

This value for the polar surface field implies that there may exist regions on the secondary star with high magnetic field, i.e star spots or prominences, where electrons can be trapped. Also, it has been shown [11] that the region of closed field lines of the secondary star's magnetosphere, i.e. the so-called dead-zone, extends as far as the total binary separation, i.e.

$$
\frac{r}{a}=0.065\left(\frac{P_{\text {orb }}}{h}\right)+1,
$$

where $r$ represents the dead-zone radius, and $a$ the binary separation. This implies that for the AM Her type systems the secondary star's magnetic field in the equatorial zone can envelope the white dwarf, which may result in interesting possibilities for continuous magnetic interaction and resultant particle acceleration. For example it has been shown [80] that any disturbance of the magnetospheric flux tubes of the secondary star in close binaries can account for field aligned potentials and currents, where particles can be accelerated in zones of low conductivity. These authors have shown that these potentials, for the parameters selected, can reach values of (using Eq. 2.11)

$$
\begin{aligned}
\Phi_{\|} & =\left(\frac{300\left(\Delta B_{\perp}\right)^{3}}{64 \pi e n^{3 / 2} \sqrt{4 \pi k T}}\right) \text { Volt } \\
& \sim 1\left(\frac{\Delta B_{\perp}}{0.5 B_{\circ}}\right)^{3}\left(\frac{n_{\mathrm{e}}}{10^{11} \mathrm{~cm}^{-3}}\right)^{-3 / 2}\left(\frac{T_{2}}{10^{5} \mathrm{~K}}\right)^{-1 / 2} \mathrm{MV},
\end{aligned}
$$

where $B_{\circ} \sim 1000 \mathrm{G}$ represents the average polar value of the secondary stars's surface field strength. This could certainly explain the presence of $\sim 0.5 \mathrm{MeV}$ electrons trapped in the magnetic fields of the secondary star, which could account for the properties of the observed radio emission from AM Her (e.g. [22]). This model is based on the assumption that a slight asynchronism between the rotation of a phase locked white dwarf and secondary star will gradually induce field perturbations $\left(\Delta B_{\perp} \sim B_{\circ}\right)$ over time which will manifest in episodic events of particle acceleration and nonthermal emission. Although this prediction falls within the realm of the Mestel and Spruit magnetic braking stellar wind model [81], it provides an interesting mechanism for particle acceleration and non-thermal radio emission in the magnetospheric fields of a magnetic secondary star, which seem to correlate with the observed radio emission from AM Her.

\subsection{Gamma-ray emission from novae explosions}

In March 2010 the Fermi-LAT made the first discovery of gamma-ray emission with energy $\varepsilon_{\gamma} \geq 100 \mathrm{MeV}$ from the classical nova V407 Cygni [33], which were followed later by gamma-ray detections from other classical novae, i.e. V959 Monocerotis and V1324 Scorpii in June 2012 and V339 Delphini in August 2013 (see Fig. 11). Apart from V407 Cygni, where the companion star is a red giant, the other three systems have ordinary sun-like companions. V407 Cyg is a recurrent symbiotic nova, where the red giant companion is producing a strong stellar wind, which generates a shock where the fast-moving nova ejecta interacts with the thick stellar wind. This shocked 

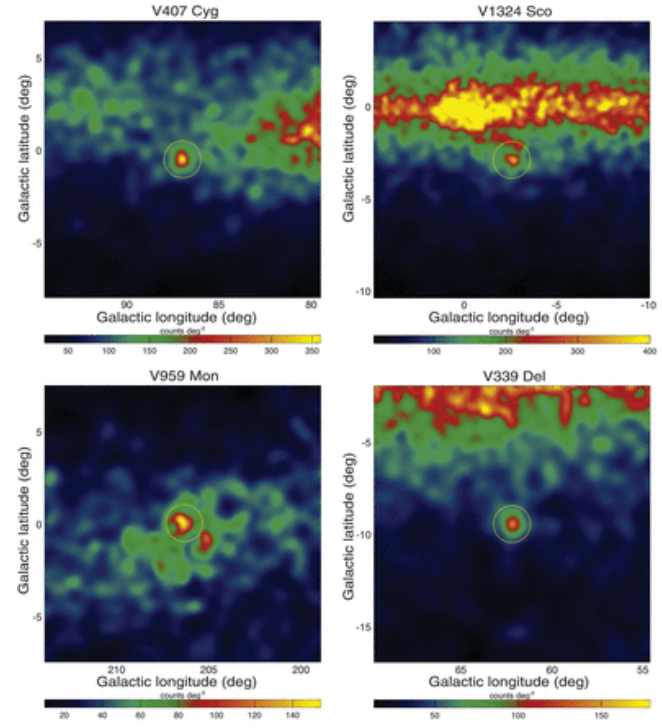

Figure 11: Fermi-LAT skymaps showing the gamma-ray detections of 4 Novae systems. Adopted from [33].

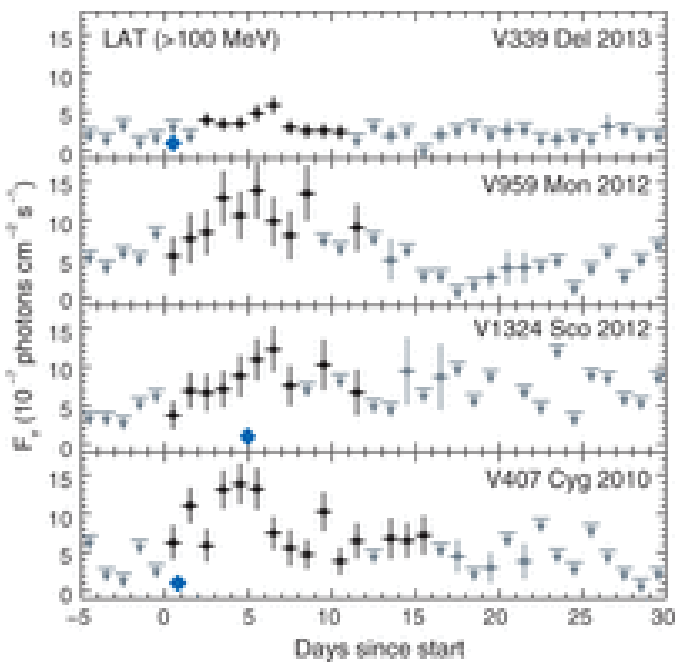

Figure 12: Gamma-ray lightcurves of the 4 Novae systems above $100 \mathrm{MeV}$. Adopted from [33].

zone may be a place where charged particles are accelerated to very high energies with associated gamma-ray production. In normal classical novae, there is no stellar wind from the companion, which poses a question regarding the mechanism responsible for the gamma-ray emission from the other three sources. Although V407 Cyg, Nova Sco 2012, and Nova Mon 2012 were different classes of events in the optical, their gamma-ray spectra are quite similar. All three have soft spectra that take several days to rise to their peaks and last 2-3 weeks (see Fig. 12). Swift X-ray observations showed no corresponding X-ray source in Nova Sco 2012, but an X-ray source was detected in Nova Mon 2012. The Very Large Array (VLA) observed both objects in the radio and found a faint detection in Nova Sco 2012. Observations of the other system, Nova Mon 2012, suggested a bi-polar structure with associated optically thick Brehmsstrahlung emission, possibly from accelerated electrons [82].

These initial Fermi-LAT gamma-ray detections were followed with another classical nova system, Nova Sgr 1015 (no 2). A Target of Opportunity (ToO) observation of the classical nova, Nova Sgr 2015 No. 2 about 2 days after the optical discovery showed preliminary evidence for $>100 \mathrm{MeV}$ emission at the 3.8 sigma significance level, with an average flux, $F(\mathrm{E}>100 \mathrm{MeV}) \sim$ $10^{-7} \mathrm{ph} \mathrm{cm}^{-2} \mathrm{~s}^{-1}$, assuming a single power-law with photon index $=2.2$. Detectable gamma-ray emission was found 1.5 days after the optical emission peaked around March 21.5, similar to V339 Del 2013 [33].

The detection of gamma-ray emission above $100 \mathrm{MeV}$ poses interesting possibilities regarding possible mechanisms for particle acceleration and associated gamma-ray emission from nonrelativistic shocks [82]. These authors have shown that the conversion rate of relativistic particles to Fermi-LAT gamma-ray emission is at most a few tens of a per cent. They also showed that the low ratio of optical to gamma-ray luminosity of two sources, i.e. V1324 Sco and V339 Del sets a limit on the fraction $\left(\alpha_{\mathrm{nth}}\right)$ of the shock power used to accelerate relativistic particles, which is 
$\alpha_{\text {nth }} \geq 10^{-2}$ and $\alpha_{\text {nth }} \geq 10^{-3}$ respectively [82]. Based on these findings, lepton based gamma-ray emission is discarded as a result of the lower electron acceleration efficiency $\alpha_{\mathrm{nth}} \sim 10^{-4}-10^{-3}$, based on galactic cosmic ray observations and particle in cell simulations [82].

The fact that the estimated number of novae per year in the Milky way galaxy is around 40 [83], combined with the improved multi-frequency sensitivity and resolution of future telescopes like the SKA precurser meerKAT and the Cerenkov Telescope Array (CTA), may yet provide many more detections of these enigmatic events, which will certainly throw light on the possible gammaray production mechanism during these events.

\section{Discussion}

An attempt has been made to give a unified discussion focussing on the role of magnetic fields in close binary systems like the cataclysmic variables to drive transient emission processes from radio to VHE gamma-rays. It has been shown that for magnetic accretors the disc outside the corotation radius drains less effectively than the region inside the corotation radius as a result of the centrifugal effects of the frozen-in magnetic field of the accreting source. This leads to a build-up of disc and magnetic field outside the corotation radius which releases flare events in terms of a gravito-magnetic instability, leading to episodes of high mass accretion and possibly ejection of plasma from the accretion disc. In the event of magnetized plasma, it may lead to synchrotron emitting clouds that may be detectable with sophisticated radio telescope networks like the SKA precurser meerKAT that is currently under construction in South Africa. Furthermore, a discussion has been presented of particle acceleration processes in magnetized plasmas that can drive transient events of particle acceleration and non-thermal emission in the cataclysmic variables. It has specifically been applied to explain the reported non-thermal emission in the propeller driven nova-like variable AE Aquarii, as well as AM Her type systems (polars) that show evidence of non-thermal radio emission. The recent Fermi-LAT detections of gamma-ray emission at energies above $100 \mathrm{MeV}$ from novae, opens up new possibilities to study and constrain particle acceleration in non-relativistic shocks that form in nova outbursts. The improved sensitivity of a new generation of telescopes over the electromagnetic spectrum, like meerKAT (radio), Astrosat (X-ray) and CTA in VHE gamma-rays may prove to be the advent of a golden era in the study of these fascinating systems.

\section{Acknowledgements}

The author thank the organisers for the invitation to present this results at the conference and for the hospitality during the conference.

\section{References}

[1] M. Cropper, The Polars, Space Science Reviews 54 (1990) 195

[2] W.H.G. Lewin, J. van Paradijs \& P.J. van den Heuvel, X-Ray Binaries, Cambridge Astrophysics Series, 26 (1995) 
[3] N.U. Shakura \& R.A. Sunyaev, Black holes in binary systems. Observational appearance, A\&A 24 (1997) 337

[4] F. Meyer \& E. Meyer-Hofmeister, On the Elusive Cause of Cataclysmic Variable Outbursts, $A \& A 104$ (1981) L10

[5] A. Ichikawa \& Y. Osaki, Time evolution of the accretion disk radius in a dwarf nova, PASJ 44 (1992) 15

[6] E.P. Velikhov, Stability of an ideally conducting liquid flowing between cylinders rotating in a magnetic field, Sov. Phys. JETP 36 (1959) 1389

[7] S.A. Balbus \& J.F. Hawley, A powerful local shear instability in weakly magnetized disks. I - Linear analysis. II - Nonlinear evolution, ApJ 376 (1991) 214

[8] S.A. Balbus \& J.F. Hawley, Instability, turbulence, and enhanced transport in accretion disks, Rev. Mod. Phys. 70 (1998) 1-73

[9] S.A. Balbus, Enhanced Angular Momentum Transport in Accretion Disks, ARA\&A 41 (2003) 555

[10] P.J. Meintjes, Magnetized fragmented mass transfer in cataclysmic variables: AE Aquarii, a trial case, MNRAS 352 (2004) 416

[11] P.J. Meintjes \& E. Jurua, Secondary star magnetic fields in close binaries, MNRAS 372 (2006) 1279

[12] V.M. Lipunov, Astrophysics of Neutron Stars, Astronomy and Astrophysics Library, SpringerVerlag (1992)

[13] J. Patterson, The DQ Herculis Stars, PASP 106 (1994) 209

[14] O.C. de Jager, P.J. Meintjes, D. O'Donoghue \& E. Robinson, The discovery of a brake on the white dwarf in AE Aquarii, MNRAS 267 (1994) 577

[15] G.A. Wynn \& A.R. King, Diamagnetic accretion in intermediate polars - I Blob orbits and spin eveolution MNRAS 275 (1995) 9

[16] G.A. Wynn, A.R. King \& K. Horne, A magnetic propeller in the cataclysmic variable AE Aquarii MNRAS 286 (1997) 436

[17] P.J. Meintjes \& L.A. Venter, The diamagnetic blob propeller in AE Aquarii and the nonthermal radio to mid-infrared emission MNRAS 360 (2005) 573

[18] D.V. Bisikalo \& A.G. Zhilkin, Accretion processes in intermediate polars with asynchronous rotations of white dwarfs, inproceedings of Golden Age of Cataclysmic Variables and Related Objects, Palermo, (eds. F. Giovanelli \& L. Sabau-Graziati), MEMORIE S.A.It 83 (2) (2012) 562 
[19] G. Chanmugam \& G.A. Dulk, Radio Emission from AM Her type Binaries, ApJ 255 (1982) L107

[20] G. Chanmugam \& G.A. Dulk, AM Herculis Binaries: Particle Acceleration, Radio Emission and Synchronization, in Poceedings of Cataclysmic Variables and Related Objects (1983) (eds. M. Livio and G. Shaviv) (Dordrecht Reidel Publishing Company), 223

[21] G.A. Dulk, T.A. Bastian \& G. Chanmugam, Radio Emission from AM Herculis: The quiescent component and and outburst, ApJ 273 (1983) 249

[22] R.M. Hjellming Radio Stars, in Galactic and Extra-Galactic Radio Astronomy, 2nd ed. (1988) eds. G.L. Verschuur \& K.I. Kellerman, Springer-Verlag, New-York, 415-416

[23] J.A. Bookbinder \& D.Q. Lamb, Discovery of radio emission fro AE Aquarii, ApJ 323 (1987) L131

[24] T.S. Bastian, G.A. Dulk \& G. Chanmugam, Radio Flares from AE Aquarii: A Low power Analogue to Cyg X-3, ApJ 324 (1988) 431

[25] G. Dubus et al., Spitzer Space telescope Observations of the Cataclysmic variable AE Aquarii, ApJ 663 (2007) 516

[26] P.A. Mason \& C. Gray, An investigation of radio emission in magnetic cataclysmic variables, in the Proceedings of IAU Colloquium 190: Magnetic Cataclysmic Variables, ASP Conf. Series, 315 (2004) (eds. S. Vrielman \& M. Cropper), San Francisco, 237

[27] C.C.G. Bowden et al., $350 \mathrm{GeV}$ gamma-rays from AE Aquarii, Astropart. Phys. 1 (1992) 47

[28] P.J. Meintjes et al., AE Aquarii: An emitter of pulsed TeV gamma-rays resembling optical emission during flares , ApJ 401 (1992) 325

[29] P.J. Meintjes et al., Simultaneous optical and TeV gamma-ray observations of the cataclysmic variable AE Aquarii, ApJ 434 (1994) 292

[30] M.J. Lang et al. A search for TeV gamma-ray emission from AE Aquarii, Astroparticle Physics 9 (1998) 203

[31] C.W. Mauche et al., Multiwavelength campaign of observations of AE Aquarii, in Proceedings of Golden Age of Cataclysmic variables and Related Objects, Mem. S.A. It Vol 83 (2) (2012), (eds. F. Giovanelli \& L. Sabau-Graziati), 651

[32] R. López-Coto et al.. A search for TeV $\gamma$-ray emission from AE Aquarii coincident with high optical and X-ray states with the MAGIC telescopes, in Proceedings of The 33 rd international cosmic-ray conference, Rio de Janeiro 2013 arXiv:1309.2503v1 [astrp-ph.HE] 10 Sep 2013

[33] The Fermi-LAT Collaboration, Fermi establishes classical novae as a distinct class of gamma-ray sources, Science, 345 (2014) 554 
[34] C.G. Campbell, Magnetohydrodynamics in Binary Stars, Kluwer Academic Publishers (Dordrecht Boston London), (1997) 171-191

[35] J.D. Jackson, Classical Electrodynamics (2nd ed), Wiley \& Sons, New York, (1975) 473, 552

[36] A.R. Choudhuri, The physics of fluids and plasmas, Cambridge Univ. Press., Cambridge, (1998) 260-262

[37] P.J. Meintjes \& E. Breedt, Magnetic viscocity: Outbursts and outflows in accretion driven systems, in Proceedings of High Energy Astrophysics in Southern Africa: A multi-frequency perspective of new frontiers in high energy astrophysics in Southern Africa (eds. B van Soelen \& P. Meintjes) Mem. S.A. It Vol 86 (1) (2015) 89

[38] P.J. Meintjes, E. Breedt \& M. Erwee, Magnetized Accretion Discs and the Dwarf Nova Phenomenon, in Proceedings of Frontier Research in Astrophysics (eds. F. Giovanelli, J. Beall, S. Colafeancesco, D. Bisikal and L. Sabau-Graziati) Proceedings of Science (2015) POS(FRAPWS2014)010

[39] E.N. Parker, The acceleration of particles to high energy, in proceedings of The Physics of Non-Thermal Radio Sources, G.Setti (ed.), Dordrecht-Reidel Publishing Company, (1976) 137

[40] P.M. Bauleo \& J.R. Martino, The dawn of the particle astronomy era in ultra-high energy cosmic sources Nature $\mathbf{4 5 8}$ (2009) 847

[41] L. Mestel Stellar Magnetism (1999), Claredon Press Oxford, 533- 630

[42] G. Haerendel, Particle acceleration from field aligned potential droms, ApJS (1994) 90765

[43] P.J. Meintjes \& O.C. de Jager, Propeller spin-down and the non-thermal emisiion from AE Aquarii MNRAS 311 (2000), 611

[44] M.A. Raadu, The physics of double layers and their role in astrophysics, Phys. Reports $\mathbf{1 7 8}$ (1989) 25

[45] M.A. Raadu, Energy release in double layers, Space Science Reviews 68 (1994) 29

[46] R.G. Giovanelli, A theory of chromospheric flares Nature (1946) 15881

[47] F. Hoyle, Some recent advances in Solar Physics (1949), Cambridge Univ. Press, Cambridge

[48] E. Priest \& T. Forbes, Magnetic Reconnection: MHD theory and application (2000), Cambridge Univ. Press, Cambridge

[49] P.A. Sweet, Neutral point theory in solar flares, in Proceedings of IAU Symp. 6: Electromagnetic Phenomena in Cosmical Plasmas (1958) (ed. B Lehnert), Cambridge Univ. Press, 123-134

[50] P.A. Sweet, The production of high energy particles in solar flares, Nuovo Cimento suppl $\mathbf{8}$, Ser. X (1958) 188-196 
[51] E.N. Parker Sweet's mechanism for merging fields in conducting fluids J. Geophys. Research 62 (1957) 509-520

[52] H.E. Petchek, Magnetic Field Annihilation, in The Physics of Solar Flares (1964) (ed. W.N. Hess), (NASA SP-50, Washington, DC) 425-439

[53] S.I. Syrovatsky, Formation of a current sheet in a plasma with frozen-in strong magnetic fields, Sov. Phys. JETP 33 (1971) 933

[54] D. Biskamp, Hot spots in extragalactic radio sources, Lecture Notes in Physics (1988) (eds. K. Meisenheimer \& H.J Röser), Springer, Berlin 279

[55] A. Benz, Plasma Astrophysics, Astrophys. Space Science Library 184 (1994) Kluwer Academic Publishers, Dordrecht 285

[56] W.A. Baan, Neutron stars as X-ray burst sources, ApJ 214 (1977) 245

[57] F.K. Lamb, A.C. Fabian, J.C. Pringle \& D.Q. Lamb, A model for bursting x-ray sources: Time dependent accretion by magnetic neutron stars and degenerate white dwarfs, ApJ 217 (1977) 197

[58] J. Smak Eruptive binaries II U Geminorum, Acta Astronomica 21 (1971) 15

[59] J-P Lasota, The disc instability model for dwarf novae and low mass $x$-ray transients, New Astronomy Reviews (2001) (astro-ph/0102072)

[60] J. Frank, A.R. King \& D. Raine, Accretion Power in Astrophysics, 3rd ed. (2002) Cambridge Univ. Press, Cambridge, 121-128

[61] J. Li \& D.T. Wickramasinghe, On spin-up/spin-down torque reversals in disc accreting pulsars , MNRAS 300 (1998) 1015

[62] C.G. Campbell, The inner structure of an accretion disc around a magnetized neuutron star Geophys. Astrophys. Fluid Dynamics 63 (1992) 179

[63] E. Breedt, A magnetohydrodynamic perspective on magnetic viscosity and mass transfer in disc-accreting cataclysmic variable stars, M.Sc thesis, University of the Free State (UFS), Unpublished, (2005) 63

[64] W.F. Hughes \& J.A Brighton, Fluid Dynamics, 3rd ed (1999) Schaum's Outline Series, McGraw-Hill New York 249

[65] L.D. Landau \& E.M Lifshitz, Fluid Mechanics, 2nd ed (1987) Pergamon Press Oxford, 132133

[66] D. Biskamp, Magnetohydrodynamic Turbulence (2003) Cambridge University press, Cambridge 33-65

[67] B. Warner Cataclysmic variable Stars (1995) Cambridge University Press, Cambridge 126215 
[68] R.D. Blandford \& D.G. Payne, Hydromagnetic flows from accretion discs and the production of radio jets, MNRAS 199 (1982) 883

[69] M.R. Hayashi, K. Shibata \& R. Matsumoto, X-ray flares and mass outflowsdriven by magnetic interactionbetween a protostar and its surrounding disc, ApJ 468 (1996) L37

[70] H. van der Laan A model for variable extragalactic radio sources, Nature (1966) 2111131

[71] P.J. Meintjes, B Oruru \& A. Odendaal, The multi-frequency properties of AE Aquarii and its evolution form a high mass transfer history, in Proceedings of Golden Age of Cataclysmic Variables and related Objects (Palermo 2011) Mem. S.A.It. 83 (2012) 643

[72] P.J. Meintjes, A Odendaal \& H.J. van Heerden, AE Aquarii: A short review in Proceedings of Golden Age of Cataclysmic Variables and related Objects II (Palermo 2013) Acta Polytechnica CTU proceedings 2(1) (2015) 86

[73] W. Becker \& J. Trümper, The x-ray luminosity of rotation powered neutron stars, A\&A $\mathbf{3 2 6}$ (1997) 682

[74] Y. Terada et al., Suzaku discovery of hard X-ray pulsations from a rotating magnetized white dwarf, AE Aquarii, Publ. Astron. Soc. Japan 60 (2008) 387

[75] O.C. de Jager, Evidence for particle acceleration in a magnetized white dwarf from radio to gamma-ray observations ApJ Suppl. 90 (1994) 775

[76] P.J. Meintjes \& L.A. Venter, Modelling the continuous radio outbursts in AE Aquarii, MNRAS 341 (2003) 891

[77] L.A. Venter \& P.J. Meintjes, Kelvin-Helmholtz driven propeller in AE Aquarii: A Unified model for thermal and non-thermal flares, MNRAS 366 (2006) 557

[78] B. Oruru \& P.J. Meintjes, X-ray characteristics and the spectral energy distribution of AE Aquarii, MNRAS 421 (2012) 1557

[79] P. Goldreich \& D. Lynden-Bell, Io, a Jovian unipolar inductor, ApJ 156 (1969) 59

[80] L.A. Venter \& P.J. Meintjes, A tenuous X-ray corona enveloping AE Aquarii, MNRAS 378 (2007) 681

[81] L. Mestel \& H.C. Spruit, On magnetic braking of late-type stars, MNRAS 226 (1987) 57

[82] B.D. Metzger et al. Gamma-ray novae as probes of relativistic particle acceleration at nonrelativistic shocks, MNRAS, 450 (2015) 2735

[83] D. Prialnik, Novae, in Encyclopedia of Astronomy and Astrophysics (2001) London Institute of Physics Publishing and Nature Publishing Groups pp. 1846-1856 